\title{
Asymmetric non-Abelian orbifolds and model building
}

\author{
Zurab Kakushadze, ${ }^{*}$ Gary Shiu, ${ }^{\dagger}$ and S.-H. Henry Tye ${ }^{\ddagger}$ \\ Newman Laboratory of Nuclear Studies, Cornell University, Ithaca, New York 14853-5001
}

(Received 16 July 1996)

\begin{abstract}
The rules for the free fermionic string model construction are extended to include general non-Abelian orbifold constructions that go beyond the real fermionic approach. This generalization is also applied to the asymmetric orbifold rules recently introduced. These non-Abelian orbifold rules are quite easy to use. Examples are given to illustrate their applications. [S0556-2821(96)00724-2]
\end{abstract}

PACS number(s): 11.25.Hf, 11.25.Mj, 12.10.Dm

\section{INTRODUCTION}

This paper presents the generalization of the rules for the free fermionic and asymmetric Abelian orbifold constructions to include non-Abelian orbifolds. The orbifold construction [1], both Abelian and non-Abelian, is an algebraic approach to string model building. The rules presented here are particularly useful for asymmetric orbifolds [2], where the geometric approach is less powerful.

Consider a typical compactification of an $n$-dimensional space $R^{n}$. An $n$-dimensional orbifold $\Omega$ is constructed by identifying points of $R^{n}$ under a space group $S$ of rotations and translations. The subgroup of $S$ formed by pure translations is referred to as the lattice $\Lambda$, since identification of points of $R^{n}$ under $\Lambda$ defines the torus $\Gamma^{n}$. In this case, $\Omega$ can be obtained by identifying points of $\Gamma^{n}$ under the point group $P$, which is the discrete subgroup of only rotations in $S$. This may be summarized by

$$
\Omega=R^{n} / S=\Gamma^{n} / P .
$$

Of course, the lattice $\Lambda$ in a consistent string model is Lorentzian. Also, it is understood that appropriate twisted sectors must be included in the orbifold model. More generally, one may construct the same model $\Omega$ by starting with another model $\Omega^{\prime}$ modded (or orbifolded) by an appropriate group $G$ of translations and rotations. In general, there are more than one choice of $\left(\Omega^{\prime}, G\right)$ that will reproduce the same $\Omega$. For example, we may start with an appropriate torus $\bar{\Gamma}$ and identify its points under a specific group $\bar{P}$ of translations and rotations. Here, $\bar{P}$ is a subgroup of the isometry group of the torus $\bar{\Gamma}$ :

$$
\Omega=\Omega^{\prime} / G=\bar{\Gamma} / \bar{P}=\cdots .
$$

A priori, the group $G$ may be either Abelian or non-Abelian. (Here, a typical $\bar{P}$ is non-Abelian.) An orbifold that involves a (non-)Abelian $G$ is referred to as a (non-)Abelian orbifold. An asymmetric orbifold refers to an orbifold when the group actions on the left movers and on the right movers are dif-

\footnotetext{
*Present address: Lyman Laboratory, Harvard University, Cambridge, MA 02138. Electronic address: zurab@hepth.cornell.edu

†Electronic address: shiu@ hepth.cornell.edu

‡ELectronic address: tye@ @epth.cornell.edu
}

ferent. So it may happen that the same model $\Omega$ can be reached via an Abelian orbifold or via a non-Abelian orbifold, either symmetric or asymmetric, all depending on which $\Omega^{\prime}$ one starts with.

Let us recall the situation with the free fermionic string model construction [3]. A particular free fermionic string model is dictated by the choice of spin structures and their correlations. Since the spin structures are generically leftright asymmetric, these models are asymmetric orbifolds in the orbifold language. However, the present rules for the free fermionic string construction do not allow general types of non-Abelian orbifolds. This is unnecessarily restrictive, since in this construction, we must always start from a unique $\Omega^{\prime}$. For example, in the four-dimensional heterotic string case, $\Omega^{\prime}$ is simply the $\mathrm{SO}(44)$ tachyonic string model. Although the set of spin structures is obviously commuting, in the real world-sheet fermionic basis, they do yield models that correspond to a special type of non-Abelian orbifolds (that involve noncommuting $\mathbf{Z}_{2}$ twists) in the usual orbifold language. However, the present rules do not allow the construction of other types of non-Abelian orbifolds. A simple example will illustrate this point.

Consider the $\mathbf{Z}_{2}$ orbifold of a single compactified boson, which can have arbitrary radius. However, if we use the present free fermionic string construction, we must start with the boson at radius 1 . The rules allow us to construct either (1) a $\mathbf{Z}_{2}$ twisted boson at radius 1 by giving the two real world-sheet fermions different spin structures (one periodic and the other antiperiodic), or (2) a boson with radius at any rational value, by giving the single complex fermion appropriate spin structures.

But the present free fermionic string rules do not permit the construction of a twisted boson at a radius away from 1, since the two sets of spin structures mentioned Above are not compatible with each other. In the orbifold language, the above construction involves $G=\bar{P}$ which is non-Abelian.

Fortunately, the generalization of the rules needed is not difficult to obtain. In this paper, we shall extend the free fermionic construction rules to include orbifold actions of non-Abelian groups. Some of the important steps in reaching our rules are already developed in the literature. In addition to the original papers by Dixon, Harvey, Vafa, and Witten [1], we refer the reader to the work by Ginsparg [4], and that by $\mathrm{Li}$ and Lam [5]. We believe the rules presented in this paper are relatively easy to use, especially for intricate models. With these new rules, we expect the free fermionic con- 
struction to be closer to the usual orbifold constructions. This should not be surprising, since the equivalence of worldsheet fermions and bosons is well known.

Recently, the free fermionic string construction rules have been generalized to include world-sheet bosons [6]. These relatively simple rules are useful in the construction of asymmetric orbifolds, since they allow us to consider twists other than $\mathbf{Z}_{2}$ twists. In this construction, the starting $\Omega^{\prime}$ is quite flexible. Generically, it is chosen to be an $N=4$ supersymmetric Narain model [7]. The generalization of these rules to include non-Abelian orbifolds is essentially identical to that for the free fermionic case. Using these rules, it is quite straightforward to construct general non-Abelian orbifolds, including models with non-Abelian point groups. The reader who is interested only in the bosonic formulation may go directly to Sec. V, then to Sec. III and VI.

We shall start with the free fermionic string construction. In Sec. II, we briefly review its rules. In Sec. III, we give the generalized rules for the construction of consistent nonAbelian orbifold models within the framework of the free fermionic string construction. In Sec. IV, we illustrate these rules by giving some explicit examples. We consider examples in both ten and four dimensions. In Sec. V, we review the rules for asymmetric orbifolds. The generalization of these rules to the non-Abelian case is identical to that given for the free fermionic case in Sec. III. To illustrate the use of the rules for non-Abelian asymmetric orbifold constructions, we give two examples in Sec. VI. The first example involves a non-Abelian point group $P$, namely, the permutation group $S_{3}$, plus Wilson lines. The second example is the three-family $\mathrm{SO}(10)_{3}$ model recently constructed [8]. Here, we recast that model as a non-Abelian orbifold. Appendix gives a discussion of the $\mathbf{Z}_{2}$ orbifold of a single compactified boson.

\section{PRELIMINARIES}

In this section, we briefly review the rules for constructing free fermionic string models as presented in Ref. [9]. To be concrete, let us focus on heterotic strings compactified to four space-time dimensions. In the light-cone gauge which we adopt, the world-sheet degrees of freedom consist of two string coordinates and 10 (22) right- (left)-moving chiral world-sheet complex fermions. A string model is succinctly defined by the choice of spin structures, i.e., a set of basis vectors $\left\{V_{0}, V_{1}, \ldots, V_{n}\right\}$. Each $V_{i}$ is a 32 (rational) component vector with Lorentzian signature $\left[(-1)^{10},(+1)^{22}\right]$. Let $\alpha_{i} V_{i}$ and $\beta_{i} V_{i}$ be two linear combinations that specify the boundary conditions of the 32 complex fermions $\psi^{\ell}$, $\ell=1,2, \ldots, 32$ on the torus:

$$
\begin{aligned}
& \psi^{\ell}\left(\sigma_{1}+2 \pi, \sigma_{2}\right)=-e^{-2 \pi i \alpha_{i} V_{i}^{\ell}} \psi^{\ell}\left(\sigma_{1}, \sigma_{2}\right), \\
& \psi^{\ell}\left(\sigma_{1}, \sigma_{2}+2 \pi\right)=-e^{-2 \pi i \beta_{i} V_{i}^{\ell}} \psi^{\ell}\left(\sigma_{1}, \sigma_{2}\right) .
\end{aligned}
$$

It is convenient to choose $-\frac{1}{2} \leqslant V_{i}^{\ell}<\frac{1}{2}$, and we define $\overline{\alpha V}=\alpha V-\Delta(\alpha)$ where $\Delta^{l}(\alpha) \in \mathbf{Z}$, so that $-\frac{1}{2} \leqslant \overline{\alpha V}^{\ell}<\frac{1}{2}$. Consistency requires the presence of $V_{0}=\left[-\frac{1}{2}\left(-\frac{1}{2}-\frac{1}{2}\right.\right.$ $\left.-\frac{1}{2}\right)^{3} \mid\left(-\frac{1}{2}\right)^{22}$ ] where the first component labels the boundary condition of the superpartner of the two right-moving transverse space coordinates.

We impose three consistency requirements on the oneloop string partition function $Z$.

(1) World-sheet supersymmetry, i.e., the triplet constraint on each vector $V_{i}$,

$$
\begin{aligned}
s_{i} & \equiv V_{i}^{1}=V_{i}^{2+3 n}+V_{i}^{3+3 n}+V_{i}^{4+3 n} \quad(\bmod 1), \quad n=0,1,2 \\
& =0 \text { or }-\frac{1}{2} .
\end{aligned}
$$

This ensures the proper boundary conditions for the worldsheet supercurrent and hence space-time Lorentz invariance in the covariant gauge.

(2) One-loop modular invariance.

(3) Physically sensible projection, i.e., every space-time degree of freedom contributes to the partition function with the proper weight, +1 for a space-time boson [with $\alpha s=0$ $(\bmod 1)]$ and -1 for a space-time fermion [with $\alpha s=-1 / 2(\bmod 1)]$.

A consistent string model is defined by the set $\left\{V_{0}, V_{1}, \ldots, V_{n}\right\}$ and the structure constants $\left\{k_{i j}: i, j=0,1, \ldots, n\right\}$ which satisfy

$$
\begin{gathered}
k_{i j}+k_{j i}=V_{i} \cdot V_{j} \quad(\bmod 1), \\
m_{j} k_{i j}=0 \quad(\bmod 1), \\
k_{i i}+k_{i 0}+s_{i}-\frac{1}{2} V_{i} \cdot V_{i}=0 \quad(\bmod 1),
\end{gathered}
$$

where all the dot products are defined with Lorentzian signature, i.e., left movers minus right movers. Here, $m_{j}$ is the smallest positive integer such that $m_{i} V_{i}^{\ell} \in \mathbf{Z}$ for all $\ell$.

The world-sheet fermionic contribution to the one-loop partition function is then given by

$$
\begin{aligned}
Z= & \frac{1}{\Pi_{i} m_{i}} \sum_{\alpha, \beta} \operatorname{Tr}\left\{(-1)^{\alpha s} q^{H} \frac{L}{\alpha v} q^{H \frac{R}{\alpha V} \exp }\right. \\
& \left.-2 \pi i \beta_{i}\left(V_{i} \cdot N_{\overline{\alpha V}}-\sum k_{i j} \alpha_{j}-s_{i}-V_{i} \cdot \overline{\alpha V}\right)\right\},
\end{aligned}
$$

where $\alpha s \in \mathbf{Z}\left(\mathbf{Z}+\frac{1}{2}\right)$ for space-time bosons (fermions). The partition function is divided into different sectors labeled by the vectors $\bar{\alpha} V$, with Hamiltonian $H_{\overline{\alpha V}}^{L}\left(H_{\overline{\alpha V}}^{R}\right)$ for the left (right) movers, and a vector of fermion number operators $N \overline{\alpha V}$ for the 32 complex fermions. A precise definition of $N_{\overline{\alpha V}}$ is given in Ref. [9]. The states that are kept in $Z$ satisfy

$$
V_{i} \cdot N_{\overline{\alpha V}}=\sum_{j} k_{i j} \alpha_{j}+s_{i}-V_{i} \cdot \overline{\alpha V} \quad(\bmod 1) .
$$

To establish a dictionary between the present construction and the orbifold approach, let us rewrite the partition function as 


$$
Z=\sum_{\alpha} \operatorname{Tr}\left((-1)^{\alpha s} q^{H} \frac{L}{\alpha v} \frac{q^{H}}{\alpha v} \prod_{i=0}^{n} P_{\alpha V}^{i}\right)
$$

where the sum is over all the sectors $\overline{\alpha V}$ and

$$
\begin{aligned}
P_{\alpha V}^{i}= & \frac{1}{m_{i}} \sum_{\beta_{i}=0}^{m_{i}-1} \exp \left[-2 \pi i \beta_{i}\left(V_{i} \cdot N \overline{\alpha V}-\sum k_{i j} \alpha_{j}\right.\right. \\
& \left.\left.-s_{i}-V_{i} \cdot \overline{\alpha V}\right)\right]
\end{aligned}
$$

are projection operators. The states that survive in $Z$ are invariant under all the projections $P_{\alpha V}^{i}$ On the other hand, the partition function of an orbifold by the action of a discrete Abelian group $G$ is

$$
Z=\sum_{g \in G} \operatorname{Tr}\left[(-1)^{F_{g}} q^{H_{g}^{L}} \bar{q}_{g}^{R} P_{g}\right]=\frac{1}{|G|} \sum_{g, h \in G} Z(g, h)
$$

where

$$
P_{g}=\frac{1}{|G|} \sum_{h \in G} h .
$$

Therefore, for each model constructed from complex worldsheet fermions, we can associate an Abelian orbifold with orbifold group $G=\mathbf{Z}_{m_{0}} \times \mathbf{Z}_{m_{1}} \times \cdots \times \mathbf{Z}_{m_{n}}$ (where $\mathbf{Z}_{m_{0}}$ is always $\mathbf{Z}_{2}$ ). To make the correspondence more explicit, we denote $V_{g}=\overline{\alpha V}$ and $V_{h}=\overline{\beta V}$. The $\mathbf{0}$ sector $(\overline{\alpha V}=0)$ is the identity sector while all the others are the twisted (or shifted) sectors.

As is clear from the rules, given the orbifold group $G$, there are distinct choices of the structure constants (or torsions) $\left\{k_{i j}\right\}$, corresponding to different choices of projections in the various sectors. If the boundary conditions are always periodic or antiperiodic, the complex fermion may be split into two real fermions, which may then have different boundary conditions. When we allow real fermions with different boundary conditions, the set $\left\{V_{i}\right\}$ must satisfy the cubic constraint

$$
4 \sum_{l: \text { real }} V_{i}^{l} V_{j}^{l} V_{k}^{l}=0 \quad(\bmod 1) \text { for all } i, j, k .
$$

This implies that we can find a complex fermion basis for any three-vectors. Typically, there is no complex fermion basis for the complete set $\left\{V_{i}\right\}$. In order to stay within the framework of complex world-sheet fermions, one has to introduce the notion of non-Abelian orbifolds. As we shall see, the free fermionic string model construction covers only a special type of non-Abelian orbifolds. In the next section, we will further generalize the above formulation to include other types of non-Abelian orbifolds.

\section{NON-ABELIAN ORBIFOLDS}

We are now ready to discuss the general case of nonAbelian orbifolds $[1,4,5]$. Starting with a consistent string model, whose Hilbert space admits a discrete symmetry $G$, modding out the theory by the action of $G$ generally results in a new string model. As discussed before, the consistency conditions for Abelian $G$ can be expressed in terms of $\left\{k_{i j}, V_{i}\right\}$. In this section, we will examine the constraints when $G$ is non-abelian. Our approach follows that of Ref. [5]. The main improvement is the inclusion of a careful treatment of the compactified six dimensions here, and the generalization to the bosonic formulation later.

It turns out that the consistency constraints can be inferred from the associated Abelian orbifolds $Z\left(G_{a}\right)$ where $G_{a}$ are Abelian subgroups of $G$. To see this, let us go back to Eq. (2.10). For Abelian $G$, the operator interpretation of Eq. (2.10) is clear. The Hilbert space decomposes into a set of twisted (and/or shifted) sectors labeled by $g$, and in each sector there is a projection onto $G$-invariant states. In taking the trace, we can always find a basis that is simultaneously diagonal in $H_{g}^{L}$ and $H_{g}^{R}$ and $h$. The projection in the case of non-Abelian $G$ requires a more careful treatment. In each twisted sector $g$, the sum over $h \in G$ includes only elements that commute with $g$. The group action, however, mixes states in conjugate sectors. This follows because, if

$$
g \psi^{\ell} g^{-1}=-e^{-2 \pi i \overline{\alpha V^{\prime}}} \psi^{\ell},
$$

then, for any $c \in G$,

$$
\left(c g c^{-1}\right)\left(c \psi^{\prime} c^{-1}\right)\left(c g c^{-1}\right)^{-1}=-e^{-2 \pi i \overline{\alpha V}}\left(c \psi c^{-1}\right) .
$$

As a result,

$$
Z(g, h)=Z\left(c g c^{-1}, c h c^{-1}\right) .
$$

The partition function may be expressed in terms of conjugacy classes $C_{i}$ :

$$
\begin{aligned}
Z(G) & =\frac{1}{|G|} \sum_{g \in G} \sum_{g h=h g} \operatorname{Tr}\left[(-1)^{F_{g}} q^{H_{g}^{L}} q^{H_{g}^{R} h}\right] \\
& =\sum_{i} \frac{1}{\left|N_{i}\right|} \sum_{h \in N_{i}} \operatorname{Tr}\left[(-1)^{F C_{i}} q^{H_{C_{i}}^{L}} \bar{q}^{\left.H_{C_{i}}^{R} h\right],}\right.
\end{aligned}
$$

where the group $N_{i}$ is the stabilizer (or little) group of the conjugacy class $C_{i}$ and is defined only up to conjugation. In general, the summation in Eq. (3.4) decomposes into distinct modular orbits, i.e., distinct subsets each of which is modular invariant. However, individual modular orbit does not satisfy physically sensible projection. In the full summation in Eq. (3.4), physically sensible projection is explicitly satisfied since in each sector labeled by $C_{i}$, the summation over $h$ is a properly normalized projection onto states invariant under the stabilizer group $N_{i}$. To see that modular invariance is satisfied, let us consider $G_{a}$ (the maximal Abelian subgroups of $G), G_{a b}=G_{a} \cap G_{b}$ and $G_{a b c}=G_{a} \cap G_{b} \cap G_{c}$, etc. It is easy to see that $Z(G)$ may be rewritten as a weighted sum of the Abelian orbifolds $Z\left(G_{a}\right), Z\left(G_{a b}\right), Z\left(G_{a b c}\right), \ldots$ :

$$
\begin{aligned}
Z(G)= & \frac{1}{|G|}\left\{\sum_{a}\left|G_{a}\right| Z\left(G_{a}\right)-\sum_{a<b}\left|G_{a b}\right| Z\left(G_{a b}\right)\right. \\
& \left.+\sum_{a<b<c}\left|G_{a b c}\right| Z\left(G_{a b c}\right)-\cdots\right\},
\end{aligned}
$$


where $Z\left(G_{a}\right)=\Sigma_{g, h \in G_{a}} Z(g, h)$. Since each of the $Z\left(G_{a} \ldots\right)$ is a modular-invariant partition function, modular invariance of $Z(G)$ is automatic. Notice that the coefficients in Eq. (3.5) always add up to one. Each partition function is properly normalized; this guarantees that the final partition function $Z(G)$ contains exactly one graviton.

Nevertheless, the above reasoning does not imply that all non-Abelian orbifolds obtained this way are consistent. Notice that each Abelian orbifold $Z\left(G_{a}\right)$ is specified by $\left\{k_{i j}^{a}, V_{i}^{a}\right\}$ [as well as the others $Z\left(G_{a b}\right), \ldots$ ]. For a given $G_{a}$ (i.e., the set $\left\{V_{a}\right\}$ ), there are, in general, inequivalent $Z\left(G_{a}\right)$ models depending on the choices of $\left\{k_{i j}^{a}\right\}$. In order to determine Eq. (3.5) precisely, we must find the appropriate $\left\{k_{i j}^{a}\right\}$ for each $Z\left(G_{a}\right)$. It turns out that the non-Abelian group properties of $G$ impose stringent constraints on the choices of $k_{i j}^{a}$ A consistent non-Abelian orbifold model exists only if all the $k_{i j}$ 's satisfy all the constraints. These constraints are divided into four types:

(1) Between different Abelian orbifolds $Z\left(G_{a}\right)$ and $Z\left(G_{b}\right)$. Consider the $g$-twisted sector in $Z\left(G_{a}\right)$. Suppose there is $c \in G$ such that $c g c^{-1}$ is outside $G_{a}$, say $c g c^{-1}$ belongs to $G_{b}$. Since $g$ and $\mathrm{cgc}^{-1}$ belong to the same conjugacy class, we have from Eq. (3.3), $Z_{a}(g, h)=Z_{b}\left(c g c^{-1}, c h c^{-1}\right)$, i.e., $Z\left(G_{a}\right)$ and $Z\left(G_{b}\right)$ are isomorphic. With an appropriate choice of the bases $\left(V_{i}^{a}=V_{i}^{b}\right)$, we have the constraints relating $Z\left(G_{a}\right)$ and $Z\left(G_{b}\right)$,

$$
k_{i j}^{a}=k_{i j}^{b} .
$$

We shall impose this constraint in the outset.

(2) Within the same Abelian orbifold $Z\left(G_{a}\right)$. Next, suppose that the isomorphism is inner, i.e., both $g, \mathrm{cgc}^{-1}$ $\in G_{a}$. Let $g_{i} \in G_{a}$ be the generators of $G_{a}$ : i.e.,

$$
V_{g_{i}}=V_{i} \quad \text { and } V_{c g c^{-1}}=\overline{\lambda_{i l} V_{l}} \text {. }
$$

Let us consider specific terms in $Z\left(G_{a}\right)$. From Eq. (3.3), $Z_{a}(g, h)=Z_{a}\left(c g c^{-1}, c h c^{-1}\right)$, where

$$
V_{g}=\overline{\alpha V}, \quad V_{h}=\overline{\beta V}, \quad V_{c g c^{-1}}=\overline{\alpha \lambda V}, \quad V_{c h c^{-1}}=\overline{\beta \lambda V} .
$$

Since these two sectors are isomorphic, if we confine ourselves to the $N_{\overline{\alpha \nu}}=N_{\overline{\alpha \lambda V}}=0$ states in the trace, we have

$$
\begin{aligned}
& \exp \left[2 \pi i \beta_{i}\left(\sum k_{i j} \alpha_{j}+s_{i}-V_{i} \cdot \overline{\alpha V}\right)\right] \\
& \quad=\exp \left[2 \pi \beta_{i} \lambda_{l}^{i}\left(\sum k_{l n} \lambda_{n}^{j} \alpha_{j}+s_{i}-V_{l} \cdot \overline{\alpha \lambda V}\right)\right] .
\end{aligned}
$$

Choosing $V_{g}=V_{j}, V_{h}=V_{i}$, we obtain the constraint within $\left\{k_{i j}, V_{i}\right\}_{a}$ :

$$
k_{i j}=\lambda_{i l} k_{l n} \lambda_{n j}-\Delta_{i} \cdot \lambda_{j n} V_{n}(\bmod 1),
$$

where $\Delta_{i}=\lambda_{i l} V_{l}-\overline{\lambda_{i l} V_{l}}$. Notice that $s_{i}=\lambda_{i l} s_{l}(\bmod 1)$ since conjugation does not mix space-time bosons and fermions.

(3) Between $Z\left(G_{a}\right)$ and $Z\left(G_{a b}\right)$. Then, we need to find the relations between $\left\{k_{i j}, V_{i}\right\}_{a}$ and $\left\{k_{l n}, V_{l}\right\}_{a b}$ where $G_{a b}$ is a subgroup of $G_{a}$. Clearly, we can write, for each generator $g \in G_{a b}$,

$$
V_{l}^{a b}=\overline{\gamma_{l i} V_{i}^{a}}
$$

and

$$
\begin{aligned}
k_{l n}^{a b}+k_{n l}^{a b} & =V_{l}^{a b} \cdot V_{n}^{a b} \\
& =\overline{\gamma_{l i} V_{i}^{a}} \cdot \overline{\gamma_{n j} V_{j}^{a}} \\
& =\left(\gamma_{l} V^{a}-\Delta_{l}\right)\left(\gamma_{n} V^{a}-\Delta_{n}\right) \\
& =\gamma_{l}^{i} \gamma_{n}^{j}\left(k_{i j}^{a}+k_{j i}^{a}\right)-\Delta_{l} \cdot \gamma_{n} V^{a}-\Delta_{n} \cdot \gamma_{l} V^{a} .
\end{aligned}
$$

Consistency with Eq. (3.7) gives

$$
k_{l n}^{a b}=\gamma_{l}^{i} k_{i j}^{a} \gamma_{n}^{j}-\Delta_{l} \cdot \gamma_{n} V^{a} .
$$

(4) Between three different Abelian orbifolds $Z\left(G_{a}\right)$, $Z\left(G_{b}\right)$, and $Z\left(G_{c}\right)$. Consider an element $g$ that is in $G_{a}$, $G_{b}$, and $G_{c}$. Suppose $h_{1} \in G_{a}, h_{2} \in G_{b}$. To obtain nontrivial constraints, $\quad h_{1} h_{2} \notin G_{a}, G_{b}$, i.e., $\left[h_{1} h_{2}, h_{1}\right] \neq 0$, $\left[h_{1} h_{2}, h_{2}\right] \neq 0$. Let $\quad h_{1} h_{2} \in G_{c} \quad$ and $\quad V_{g}=\overline{\alpha^{a} V^{a}}$ $=\overline{\alpha^{b} V^{b}}=\overline{\alpha^{c} V^{c}}$. Although the same $g$-twisted sector is in $Z\left(G_{a}\right), Z\left(G_{b}\right)$, and $Z\left(G_{c}\right)$, it is expressed in different bases. Fortunately, the operators that relate these different bases commute with $q^{H_{g}^{L}} \bar{q}_{g}^{R}$. If we restrict ourselves to the $N \overline{\alpha^{a} V^{a}}=N \overline{\alpha^{b} V^{b}}=N \overline{\alpha^{c} V^{c}}=0$ states, the projection operators $h_{1}, h_{2}$, and $h_{1} h_{2}$ are purely phases, and their group relation yields

$$
\begin{aligned}
\exp \{ & 2 \pi i\left[\beta_{i}^{a}\left(V_{i}^{a} \cdot \overline{\alpha^{a} V^{a}}-\sum k_{i j}^{a} \alpha_{j}^{b}-s_{i}^{a}\right)\right. \\
& \left.\left.+\beta_{i}^{b}\left(V_{i}^{b} \cdot \overline{\alpha^{b} V^{b}}-\sum k_{i j}^{b} \alpha_{j}^{b}-s_{i}^{b}\right)\right]\right\} \\
= & \exp \left[2 \pi i \beta_{i}^{c}\left(V_{i}^{c} \cdot \overline{\alpha^{c} V^{c}}-\sum k_{i j}^{c} \alpha_{j}-s_{i}^{c}\right)\right] .
\end{aligned}
$$

Since $\beta^{a} s^{a}+\beta^{b} s^{b}=\beta^{c} s^{c}(\bmod 1)$, we have the relation between $\left\{k_{i j}, V_{i}\right\}_{a},\left\{k_{i j}, V_{i}\right\}_{b}$, and $\left\{k_{i j}, V_{i}\right\}_{c}$ :

$$
\begin{aligned}
& \beta_{i}^{a}\left(V_{i}^{a} \cdot \overline{\alpha^{a} V^{a}}-k_{i j}^{a} \alpha_{j}^{a}\right)+\beta_{i}^{b}\left(V_{i}^{b} \cdot \overline{\alpha^{b} V^{b}}-k_{i j}^{b} \alpha_{j}^{b}\right) \\
& \quad=\beta_{i}^{c}\left(V_{i}^{c} \cdot \overline{\alpha^{c} V^{c}}-k_{i j}^{c} \alpha_{j}\right) \quad(\bmod 1),
\end{aligned}
$$

where $V_{g}=\overline{\alpha^{a} V^{a}}=\overline{\alpha^{b} V^{b}}=\overline{\alpha^{c} V^{c}} \quad$ and $\quad V_{h_{1}}=\overline{\beta^{a} V^{a}}, \quad V_{h_{2}}$ $=\overline{\beta^{b} V^{b}}, V_{h_{1} h_{2}}=\overline{\beta^{c} V^{c}}$. In the examples given in the next section, we shall see that Eq. (3.9) is a stringent constraint for consistent non-Abelian orbifolds, especially when the non-Abelian orbifold is also asymmetric.

Let us summarize the steps required to construct consistent non-Abelian orbifolds: (1) Rewrite the partition function $Z$ as a sum of Abelian orbifold partition functions [Eq. (3.5)]; (2) check that the free fermionic construction rules are satisfied in each Abelian orbifold $\left\{k_{i j}, V_{i}\right\}_{A}$; (3) impose extra constraints (3.6)-(3.9) between various $\left\{k_{i j}, V_{i}\right\}_{A}$. With an appropriate choice of the basis vectors, Eq. (3.6) is satisfied automatically. 


\section{EXAMPLES}

In this section, we demonstrate how to apply our rules by constructing some explicit examples. Before we focus on four-dimensional models, let us begin our discussion with ten-dimensional examples that exhibit some of the basic features of our construction.

\section{A. Ten-dimensional models}

There are only nine known consistent heterotic string models in ten dimensions [10]. Among them, eight have rank 16 gauge group and can be realized in terms of complex world-sheet fermions. The ninth has gauge group a single $\mathrm{E}_{8}$ realized at level 2, and was first constructed using real fermion basis. In the bosonic formulation, this higher-level model can be obtained by modding out the $\mathrm{E}_{8} \times \mathrm{E}_{8}$ model by the combined action of a $2 \pi$ spatial rotation and a $\mathbf{Z}_{2}$ permutation of the two $\mathrm{E}_{8}$ 's [11]. We now illustrate how the same model can be constructed by a $D_{4}$ orbifold using the rules just given. We are using this well-known example to clarify our notation.

The group $D_{4}$ is generated by two noncommuting elements $r$ and $\theta$ with the defining relations,

$$
r^{2}=\theta^{4}=1, \quad r \theta^{3}=\theta r .
$$

It has eight elements and is divided into five conjugacy classes: $C_{1}=\{1\}, C_{\theta^{2}}=\left\{\theta^{2}\right\}, C_{r}=\left\{r, r \theta^{2}\right\}, C_{r \theta}=\left\{r \theta, r \theta^{3}\right\}$, and $C_{\theta}=\left\{\theta, \theta^{3}\right\}$. The stabilizer groups are given by $N_{1}=N_{\theta^{2}}=D_{4}, N_{r}=\left\{1, \theta^{2}, r, r \theta^{2}\right\}, N_{r \theta}=\left\{1, \theta^{2}, r \theta, r \theta^{3}\right\}$, and $N_{\theta}=\left\{1, \theta, \theta^{2}, \theta^{3}, \theta^{4}\right\}$. It is straightforward to express the full partition function as a sum of Abelian orbifolds:

$$
Z=\frac{1}{2} Z_{\theta}+\frac{1}{2} Z_{r}+\frac{1}{2} Z_{r \theta}-\frac{1}{2} Z_{\theta^{2}}
$$

where $Z_{\theta}$ is a $\mathbf{Z}_{4}\left(\cong N_{\theta}\right.$ ) orbifold, $Z_{r}$ and $Z_{r \theta}$ are $\mathbf{Z}_{2} \times \mathbf{Z}_{2}$ ( $\cong N_{r}$ and $N_{r \theta}$, respectively) orbifolds, $Z_{\theta^{2}}$ is a $\mathbf{Z}_{2}$ $\left(\cong\left\{1, \theta^{2}\right\}\right.$ ) orbifold.

We have not specified the representations of the internal fermions under the action of $D_{4}$. There are five irreducible representations of $D_{4}$ : four one-dimensional representations defined by $\theta= \pm 1, r= \pm 1$ and only one two-dimensional representation:

$$
\theta=\left(\begin{array}{cc}
i & 0 \\
0 & -i
\end{array}\right), \quad r=\left(\begin{array}{ll}
0 & 1 \\
1 & 0
\end{array}\right)
$$

where we have chosen the basis such that $\theta$ is diagonal.

Let us start with the simplest model, i.e., the model generated by a single basis vector $V_{0}=\left[\left(-\frac{1}{2}\right)^{4} \mid\left(-\frac{1}{2}\right)^{16}\right]$. This model is nonsupersymmetric with 32 tachyons and gauge group $\mathrm{SO}(32)$. We may define the $D_{4}$ action by embedding eight copies of the two-dimensional representation on the left movers while leaving the right movers untouched. In other words, the left movers transform under $\theta$ and $r$ as

$$
\begin{gathered}
\theta: \psi^{2 \ell-1} \rightarrow i \psi^{2 \ell-1}, \\
\psi^{2 \ell} \rightarrow-i \psi^{2 \ell},
\end{gathered}
$$

$$
\begin{gathered}
r: \psi^{2 \ell-1} \rightarrow \psi^{2 \ell}, \\
\psi^{2 \ell} \rightarrow \psi^{2 \ell-1}
\end{gathered}
$$

for $\ell=1, \ldots, 8$. The generating vectors when expressed in their own diagonal bases are

$$
\begin{aligned}
& V_{\theta}=\left[0^{4} \mid\left(\frac{1}{4}-\frac{1}{4}\right)^{8}\right], \\
& V_{r}=\left[0^{4} \mid\left(-\frac{1}{2} 0\right)^{8}\right], \\
& V_{r \theta}=\left[0^{4} \mid\left(-\frac{1}{2} 0\right)^{8}\right], \\
& V_{\theta^{2}}=\left[0^{4} \mid\left(-\frac{1}{2}\right)^{16}\right] .
\end{aligned}
$$

We now examine the Abelian models in some detail. To begin, let us consider the $Z_{r}$ model generated by the Abelian set $\left\{V_{0}, V_{\theta^{2}}, V_{r}\right\}$. The matrix of dot products $V_{i} \cdot V_{j}$ and the structure constants $k_{i j}$ are given by

$$
V_{i} \cdot V_{j}=\left(\begin{array}{ccc}
3 & 4 & 2 \\
4 & 4 & 2 \\
2 & 2 & 2
\end{array}\right), \quad k_{i j}=\left(\begin{array}{ccc}
k_{00} & k_{\theta^{2} 0} & k_{r 0} \\
k_{\theta^{2} 0} & k_{\theta^{2} 0} & k_{r \theta^{2}} \\
k_{r 0} & k_{r \theta^{2}} & k_{r 0}
\end{array}\right) \text {, }
$$

where $k_{i j}=0, \frac{1}{2}(\bmod 1)$. There are six potentially massless sectors: $\mathbf{0}, V_{0}+V_{\theta^{2}}, V_{r}, V_{r}+V_{\theta^{2}}, V_{0}+V_{r}$, and $V_{0}+V_{r}$ $+V_{\theta^{2}}$. The spectrum-generating formula in the $\mathbf{0}$ sector (with vacuum energy $\left[-\frac{1}{2},-1\right]$ ) reads

$$
\begin{gathered}
\frac{1}{2} \sum_{i=1}^{4} N_{i}+\frac{1}{2} \sum_{i=1}^{16} \tilde{N}_{i}=\frac{1}{2} \quad(\bmod 1), \\
\frac{1}{2} \sum_{i=1}^{16} \widetilde{N}_{i}=0 \quad(\bmod 1), \\
\frac{1}{2} \sum_{i=1}^{8} \tilde{N}_{2 i-1}=0 \quad(\bmod 1) .
\end{gathered}
$$

The tachyons are projected out and the lowest energy states include the graviton and gauge bosons in the adjoint of $\mathrm{SO}(16) \times \mathrm{SO}(16)$. On the other hand, the $V_{0}+V_{\theta^{2}}$ sector (with vacuum energy $[0,-1]$ ) provides the accompanying superpartners if and only if $k_{r 0}=k_{r \theta^{2}}$. This follows from the constraints

$$
\begin{gathered}
\frac{1}{2} \sum_{i=1}^{4} N_{i}+\frac{1}{2} \sum_{i=1}^{16} \widetilde{N}_{i}=k_{00}+k_{\theta^{2} 0}+\frac{1}{2} \quad(\bmod 1) \\
\frac{1}{2} \sum_{i=1}^{16} \widetilde{N}_{i}=0 \quad(\bmod 1) \\
\frac{1}{2} \sum_{i=1}^{8} \tilde{N}_{2 i-1}=k_{r 0}+k_{r \theta^{2}} \quad(\bmod 1) .
\end{gathered}
$$

The gravitino $\left(\widetilde{N}_{i}=0\right.$ for all $i$ ) survives the projection if $k_{r 0}=k_{r \theta^{2}}$. In addition, we have space-time fermions in the adjoint representation of $\mathrm{SO}(16) \times \mathrm{SO}(16)$. If $k_{r 0} \neq k_{r \theta^{2}}$, the 
gravitino is projected out and the space-time fermions form the $(\mathbf{1 6}, 16)$ representation of $\mathrm{SO}(16) \times \mathrm{SO}(16)$.

There may be additional gauge bosons coming from the sectors $V_{r}$ and $V_{r}+V_{\theta^{2}}$. In the $V_{r}$ sector (with vacuum energy $\left.\left[-\frac{1}{2}, 0\right]\right)$, the spectrum-generating formula gives

$$
\begin{gathered}
\frac{1}{2} \sum_{i=1}^{4} N_{i}+\frac{1}{2} \sum_{i=1}^{16} \widetilde{N}_{i}=k_{r 0}+\frac{1}{2} \quad(\bmod 1) \\
\frac{1}{2} \sum_{i=1}^{16} \widetilde{N}_{i}=k_{r \theta^{2}} \quad(\bmod 1) \\
\frac{1}{2} \sum_{i=1}^{8} \widetilde{N}_{2 i-1}=k_{r 0} \quad(\bmod 1)
\end{gathered}
$$

Provided that $k_{r 0}=k_{r \theta^{2}}$, there are extra gauge bosons in the 128 spinor representation of the first $\mathrm{SO}(16)$. The $V_{r}+V_{\theta^{2}}$ sector differs from the $V_{r}$ sector by an interchange of leftmoving Neveu-Schwarz (NS) and Ramond (R) fermions. Therefore, it provides gauge bosons in the spinor representation of the second $\mathrm{SO}(16)$. Together with the gauge bosons from the $\mathbf{0}$ sector, they furnish the adjoint representation of $\mathrm{E}_{8} \times \mathrm{E}_{8}$.

Finally, the sectors $V_{0}+V_{r}$ and $V_{0}+V_{r}+V_{\theta^{2}}$ contribute space-time fermions in the $(\mathbf{1 2 8}, \mathbf{1})$ and $(\mathbf{1 , 1 2 8})$ representations of $\mathrm{SO}(16) \times \mathrm{SO}(16)$ for all choices of $k_{i j}$.

To summarize, modding out the nonsupersymmetric SO(32) model by $\mathbf{Z}_{2} \times \mathbf{Z}_{2}$ may result in two completely different models: a supersymmetric $\mathrm{E}_{8} \times \mathrm{E}_{8}$ model if $k_{r 0}=k_{r \theta^{2}}$ and a nonsupersymmetric $\mathrm{SO}(16) \times \mathrm{SO}(16)$ model otherwise.

The above analysis also applies to the $Z_{r \theta}$ model as it has the same generating vectors (although in different bases). The $Z_{\theta^{2}}$ model is generated by $\left\{V_{0}, V_{\theta^{2}}\right\}$ and so there are only two constraints in each sector. The $\mathbf{0}$ sector provides the graviton and gauge bosons in the adjoint of $\mathrm{SO}(32)$ while their superpartners reside in the $V_{0}+V_{1}$ sector.

What remains is $Z_{\theta}$ generated by $\left\{V_{0}, V_{\theta}\right\}$. The matrix of dot product $V_{i} \cdot V_{j}$ and the structure constants $k_{i j}$ are

$$
V_{i} \cdot V_{j}=\left(\begin{array}{cc}
3 & 0 \\
0 & 1
\end{array}\right), \quad k_{i j}=\left(\begin{array}{cc}
k_{00} & k_{\theta 0} \\
k_{\theta 0} & k_{\theta 0}+\frac{1}{2}
\end{array}\right),
$$

where $k_{i j}=0, \frac{1}{2}(\bmod 1)$. There are six potentially massless sectors: $\mathbf{0}, V_{0}+2 V_{\theta}, V_{\theta}, 3 V_{\theta}, V_{0}+V_{\theta}$, and $V_{0}+3 V_{\theta}$. The 0 sector contributes 256 gauge bosons in the adjoint representation of $\mathrm{U}(16)$. The sectors $V_{0}+V_{\theta}$ and $V_{0}+3 V_{\theta}$ together provide 240 space-time fermions. If $k_{\theta 0}=0$, the $V_{0}+2 V_{\theta}$ sector contributes the superpartners of the $\mathbf{0}$ sector. In addition, there are extra gauge bosons coming from the sectors $V_{\theta}$ and $3 V_{\theta}$ (both with vacuum energy $\left[-\frac{1}{2},-\frac{1}{2}\right]$ ) that complete the adjoint representation of $\mathrm{SO}(32)$. As a result, we simply recover the $Z_{\theta^{2}}$ model. However, if $k_{\theta 0}=\frac{1}{2}$, the gravitino is projected out and there are 240 space-time fermions from the $V_{0}+2 V_{\theta}$ sector. The massless states in the sectors $V_{\theta}$ and $3 V_{\theta}$ are projected out but the two tachyons survive the projection. The model has gauge group $\mathrm{U}(16)$.
What is the resultant $D_{4}$ orbifold? A priori, there are six possibilities corresponding to different choices of $k_{i j}$ in $Z_{r}$, $Z_{r \theta}$, and $Z_{\theta}$ (after taking into account the fact that $\left.Z_{r} \cong Z_{r \theta}\right)$. However, not all of them are consistent. The nonAbelian rules further restrict the number of possibilities to three. First consider the constraints between conjugate sec-

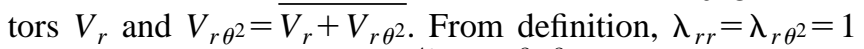
and $\Delta_{r}=V_{r}+V_{\theta^{2}}-V_{r \theta^{2}}=\left[0^{4} \mid(-1)^{8} 0^{8}\right]$. From Eq. (3.7),

$$
\begin{gathered}
k_{r r}=k_{r r}+k_{r \theta^{2}}+k_{\theta^{2} r}+k_{\theta^{2} \theta^{2}}-\Delta_{r} \cdot\left(V_{r}+V_{\theta^{2}}\right), \\
k_{r j}=k_{r j}+k_{\theta^{2} j}-\Delta_{r} \cdot V_{j}, \\
k_{j r}=k_{j r}+k_{j \theta^{2}},
\end{gathered}
$$

where $j=0, \theta^{2}$. It follows that the above equations give rise to a single constraint:

$$
k_{\theta^{2} 0}=0 .
$$

The other conjugate sectors are $\left\{V_{r \theta}, V_{r \theta^{3}}\right\}$ and $\left\{V_{\theta}, V_{\theta^{3}}\right\}$. The former gives the same constraint as above. In the latter case, notice that $V_{\theta^{3}}=\overline{3 V_{\theta}}$. Therefore, $\lambda_{\theta \theta}=3$ and $\Delta_{\theta}=\left[0^{4} \mid 1^{8}(-1)^{8}\right]$. The constraints become

$$
\begin{gathered}
k_{\theta \theta}=9 k_{\theta \theta}-\Delta_{\theta} \cdot 3 V_{\theta}=9 k_{\theta \theta}, \\
k_{\theta 0}=3 k_{\theta 0}-\Delta_{\theta} \cdot V_{0}=3 k_{\theta 0}, \\
k_{0 \theta}=3 k_{0 \theta},
\end{gathered}
$$

which are automatically satisfied.

We now turn to the constraints between three different Abelian orbifolds. Let us take $V_{h_{1}}=V_{r}, V_{h_{2}}=V_{\theta}$, and $V_{h_{1} h_{2}}=V_{r \theta}$. The common sector $V_{g}$ can be either $V_{0}$ or $V_{\theta^{2}}$. From Eq. (3.9),

$$
\begin{gathered}
V_{r} \cdot V_{0}-k_{r 0}+V_{\theta} \cdot V_{0}-k_{\theta 0}=V_{r \theta} \cdot V_{0}-k_{r \theta, 0}, \\
V_{r} \cdot V_{\theta^{2}}-k_{r \theta^{2}}+V_{\theta} \cdot \overline{2}_{\theta}-2 \mathrm{k}_{\theta \theta}=\mathrm{V}_{\mathrm{r} \theta} \cdot \mathrm{V}_{\theta^{2}}-\mathrm{k}_{\mathrm{r} \theta, \theta^{2}},
\end{gathered}
$$

which give

$$
\begin{gathered}
k_{r 0}+k_{\theta 0}=k_{r \theta, 0}, \\
k_{r \theta^{2}}=k_{r \theta, \theta^{2}} .
\end{gathered}
$$

As a consequence, the parameters $k_{i j}$ in the Abelian models are not independently chosen. If $k_{\theta 0}=0$ [i.e., $Z_{\theta}$ is the $\mathrm{SO}$ (32) model], Eq. (4.15) implies that $Z_{r}$ and $Z_{r \theta}$ both have gauge group $\mathrm{E}_{8} \times \mathrm{E}_{8}$ or $\mathrm{SO}(16) \times \mathrm{SO}(16)$. It is obvious from Eq. (4.2) that the resultant $D_{4}$ orbifold is either the $\mathrm{E}_{8} \times \mathrm{E}_{8}$ or the $\mathrm{SO}(16) \times \mathrm{SO}(16)$ model. On the other hand, if $k_{\theta 0}=\frac{1}{2}$ [i.e., $Z_{\theta}$ is the $\mathrm{U}(16)$ model], $Z_{r}$ and $Z_{r \theta}$ have gauge group $\mathrm{E}_{8} \times \mathrm{E}_{8}$ and $\mathrm{SO}(16) \times \mathrm{SO}(16)$, respectively. From Eq. (4.2),

$$
Z=\frac{1}{2} Z_{\mathrm{E}_{8} \times \mathrm{E}_{8}}+\frac{1}{2} Z_{\mathrm{SO}(16) \times \mathrm{SO}(16)}+\frac{1}{2} Z_{\mathrm{U}(16)}-\frac{1}{2} Z_{\mathrm{SO}(32)} .
$$


By simply counting the number of gauge bosons (248), space-time fermions (496) and tachyon (1) in the partition function $Z$, one can easily identify the resultant $D_{4}$ orbifold as the only rank- 8 model in ten dimensions with gauge group $\mathrm{E}_{8}$ (realized at level 2). This can be confirmed by a careful construction of the physical states.

We have seen how the rules provide nontrivial constraints between the Abelian orbifolds. There are cases in which the constraints cannot be satisfied and as a result, no consistent model can be obtained. Let us take the quaternion group $Q$ as an example [5]. It is generated by two order-4 elements $p$ and $q(p \neq q)$ with the defining relations

$$
p^{4}=q^{4}=1, \quad p^{2}=q^{2}, \quad q p=p^{3} q .
$$

There are five conjugacy classes: $C_{1}=\{1\}, C_{p^{2}}=\left\{p^{2}\right\}$, $C_{p}=\left\{p, p^{3}\right\}, C_{q}=\left\{q, q^{3}\right\}$, and $C_{p q}=\left\{p q,(p q)^{3}\right\}$. The stabilizer groups are $N_{1}=Q, \quad N_{p^{2}}=Q, \quad N_{p}=\left\{1, p, p^{2}, p^{3}\right\}$, $N_{q}=\left\{1, q, q^{2}, q^{3}\right\}$, and $N_{p q}=\left\{1, p q,(p q)^{2},(p q)^{3}\right\}$. Therefore, the full partition function is

$$
Z=\frac{1}{2} Z_{p}+\frac{1}{2} Z_{q}+\frac{1}{2} Z_{p q}-\frac{1}{2} Z_{p^{2}}
$$

The one-dimensional representations are the same as that of $D_{4}$ while the two-dimensional representation is

$$
p=\left(\begin{array}{cc}
i & 0 \\
0 & -i
\end{array}\right), \quad q=\left(\begin{array}{cc}
0 & i \\
i & 0
\end{array}\right)
$$

where we have chosen the basis such that $p$ is diagonal. Both $q$ and $p q$ take the same diagonal form as that of $p$ in their diagonal bases. Therefore, $Z_{p}, Z_{q}$, and $Z_{p q}$ are isomorphic and in what follows, we will only consider $Z_{p}$.

Again, we start with the model generated by $V_{0}$. We may embed four copies of the two-dimensional representation on the first eight left movers, leaving all the other internal fermions untouched. The generating vector $V_{p}$ is thus

$$
V_{p}=\left[0^{4} \mid\left(\frac{1}{4}-\frac{1}{4}\right)^{4} 0^{8}\right] \text {. }
$$

The matrix of dot products $V_{i} \cdot V_{j}$ and the structure constants $k_{i j}$ of $Z_{p}$ are given by

$$
V_{i} \cdot V_{j}=\left(\begin{array}{cc}
3 & 0 \\
0 & \frac{1}{2}
\end{array}\right), \quad k_{i j}=\left(\begin{array}{cc}
k_{00} & k_{p 0} \\
k_{p 0} & k_{p 0}+\frac{1}{4}
\end{array}\right),
$$

where $k_{00}, k_{p 0}=0, \frac{1}{2}(\bmod 1)$. The group multiplication [Eq. (3.9)] is not satisfied. To see this, take $g=p^{2}, h_{1}=p$, $h_{2}=q$, and $h_{1} h_{2}=p q$. On the one hand,

$$
V_{p} \cdot 2 V_{p}-2 k_{p p}+V_{q} \cdot 2 V_{q}-2 k_{q q}=0
$$

but on the other hand,

$$
V_{p q} \cdot 2 V_{p q}-2 k_{p q, p q}=\frac{1}{2} .
$$

However, it is straightforward to see that if we embed eight copies of the two-dimensional representation on the left movers, i.e.,

$$
V_{p}=\left[0^{4} \mid\left(\frac{1}{4}-\frac{1}{4}\right)^{8}\right],
$$

there are two consistent models via this $Q$ orbifold: the supersymmetric $\mathrm{SO}(32)$ model and the U(16) model.

In a similar fashion, one can show that the constraint equation (3.7) is not satisfied when the orbifold group is the extra-special $p$ group (for any odd $p \geqslant 5$ ) $[5,12]$.

\section{B. Four-dimensional models}

We have taken our exercise on ten-dimensional examples far enough; let us proceed to the more interesting fourdimensional models. A new ingredient in four dimensions is the world-sheet supersymmetry which is encoded in the triplet constraint [Eq. (2.2)].

Let us start with a free fermionic string model defined by the set of basis vectors:

$$
\begin{gathered}
V_{0}=\left[-\frac{1}{2}\left(-\frac{1}{2}-\frac{1}{2}\right)^{3} \mid\left(-\frac{1}{2}\right)^{22}\right], \\
V_{1}=\left[-\frac{1}{2}\left(\frac{1}{2} 00\right)^{3} \mid 0^{22}\right], \\
V_{2}=\left[-\frac{1}{2}\left(-\frac{1}{2} 00\right)\left(0-\frac{1}{2} 0\right)^{2} \mid\left(-\frac{1}{2}\right)^{10} 0^{10}\left(-\frac{1}{2}\right)^{2}\right], \\
V_{3}=\left[0^{7}\left(0-\frac{1}{2}-\frac{1}{2}\right) \mid 0^{10}\left(-\frac{1}{2}\right)^{2} 0^{5}\left(-\frac{1}{2}\right)^{4} 0\right] .
\end{gathered}
$$

The matrix of dot products $V_{i} \cdot V_{j}$ and the structure constants $k_{i j}$ are given by

$$
V_{i} \cdot V_{j}=\left(\begin{array}{cccc}
3 & -1 & 2 & 1 \\
-1 & -1 & -\frac{1}{2} & 0 \\
2 & -\frac{1}{2} & 2 & 0 \\
1 & 0 & 0 & 1
\end{array}\right) \text {, }
$$

$$
k_{i j}=\left(\begin{array}{cccc}
0 & 0 & 0 & 0 \\
0 & 0 & k_{21}+\frac{1}{2} & k_{31} \\
0 & k_{21} & \frac{1}{2} & k_{32} \\
0 & k_{31} & k_{32} & \frac{1}{2}
\end{array}\right) .
$$

In general, the particle content depends on the choices of $k_{i j}$. In this model, however, $\left\{k_{0 j}: j=0, \ldots, 3\right\}$ do not affect the massless spectrum and we may simply set them to zero. $V_{1}$ introduces gravitinos and hence space-time supersymmetry. $V_{2}$ serves the dual purpose of cutting the supersymmetry from $N=4$ to $N=2$, and the gauge group from $\mathrm{SO}(44)$ to $[\mathrm{SO}(20)]^{2} \times \mathrm{SO}(4) . V_{3}$ breaks the gauge group further to $\mathrm{SO}$ $(20) \times \mathrm{SO}(4) \times \mathrm{SO}(10) \times \mathrm{SO}(6) \times[\mathrm{U}(1)]^{2}$. If $k_{31}=\frac{1}{2}, V_{3}$ removes all the gravitinos; otherwise $k_{31}=0$ and the model remains $N=2$ supersymmetric.

Taking the $N=2$ supersymmetric model (i.e., choosing $k_{31}=0$ ) as our starting point, we can reduce the supersymmetry to $N=1$ as well as obtain a higher-level gauge group via non-Abelian orbifold. Again, take $D_{4}$ as an example. We define the $D_{4}$ action by choosing the generating vectors, 


$$
\begin{gathered}
V_{\theta}=\left[-\frac{1}{2}\left(0-\frac{1}{2} 0\right)\left(0-\frac{1}{2} 0\right)\left(-\frac{1}{2} 00\right) \mid\left(\frac{1}{4}-\frac{1}{4}\right)^{8}\left(-\frac{1}{2}\right)^{2}\right. \\
\left.\times 0^{2}\left(-\frac{1}{2}\right)^{2}\right], \\
V_{r}=\left[-\frac{1}{2}\left(0-\frac{1}{2} 0\right)\left(-\frac{1}{2} 00\right)\left(00-\frac{1}{2}\right) \mid\left(-\frac{1}{2} 0\right)^{8} 0^{6}\right], \\
V_{r \theta}=\left[0^{4}\left(-\frac{1}{2}-\frac{1}{2} 0\right)\left(-\frac{1}{2} 0-\frac{1}{2}\right) \mid\left(-\frac{1}{2} 0\right)^{8}\left(-\frac{1}{2}\right)^{2} 0^{2}\left(-\frac{1}{2}\right)^{2}\right], \\
V_{\theta^{2}}=\left[0^{10} \mid\left(-\frac{1}{2}\right)^{16} 0^{6}\right] .
\end{gathered}
$$

In other words, we assign two-dimensional representations only on the first 16 left movers and one-dimensional representations on the others.

Before we work out the complete spectrum, let us gain some insight from the individual Abelian models. $V_{\theta^{2}}$ does not break supersymmetry and so $Z_{\theta^{2}}$ remains $N=2$. On the other hand, both $V_{r}$ and $V_{\theta}$ break the supersymmetry to $N=1$. Using simple counting argument, physically sensible projection for the gravitinos can only be satisfied if $Z_{r \theta}$ has $N=0$ or 2 . As we shall see, $V_{r \theta}$ either removes all the gravitinos or leaves them untouched, depending on whether $k_{r \theta 1}=k_{21}$ or not.

Chiral fermions can appear only in $N=1$ models. A priori, both $Z_{\theta}$ and $Z_{r}$ may contain chiral fermions. However, in order for $Z_{\theta}$ to be part of the $D_{4}$ orbifold, $Z_{\theta}$ is necessarily nonchiral. To see this, let us assume that $Z_{\theta}$ is chiral. A particle from the $\theta$ sector together with an antiparticle from the $\theta^{3}$ sector form a single chiral multiplet. On the other hand, $\theta$ and $\theta^{3}$ belong to the same conjugacy class and hence half of the states from these two sectors are projected out. Physically sensible projection is not satisfied because the chiral multiplet cannot be divided into half.

Let us examine the Abelian models in more detail. First, consider $Z_{\theta^{2}}$ generated by the vectors $\left\{V_{0}, \ldots, V_{3}, V_{\theta^{2}}\right\}$. The matrix of dot products $V_{i} \cdot V_{j}$ and the structure constants $k_{i j}$ are given by

$$
\begin{aligned}
& V_{i} \cdot V_{j}=\left(\begin{array}{ccccc}
\cdot & & & & 4 \\
& \cdot & & & 0 \\
& & \cdot & & \frac{5}{2} \\
& & & \cdot & \frac{1}{2} \\
4 & 0 & \frac{5}{2} & \frac{1}{2} & 4
\end{array}\right), \\
& k_{i j}=\left(\begin{array}{ccccc}
\cdot & & & & k_{\theta^{2} 0} \\
& \cdot & & & k_{\theta^{2} 1} \\
& & \cdot & & k_{\theta^{2} 2}+\frac{1}{2} \\
& & & \cdot & k_{\theta^{2} 3}+\frac{1}{2} \\
k_{\theta^{2} 0} & k_{\theta^{2} 1} & k_{\theta^{2} 2} & k_{\theta^{2} 3} & k_{\theta^{2} 0}
\end{array}\right) .
\end{aligned}
$$

The gauge bosons only come from the $\mathbf{0}$ sector. With the addition of $V_{\theta^{2}}$, the gauge group is broken to $\mathrm{SO}(20) \times \mathrm{SO}(4) \times \mathrm{SO}(8) \times \mathrm{U}(1) \times \mathrm{SO}(6) \times[\mathrm{U}(1)]^{2} . \quad$ The two gravitinos in the $V_{1}$ sector are not projected out and the model remains $N=2$ supersymmetric.

Next, $Z_{r}$ is generated by $\left\{V_{0}, \ldots, V_{3}, V_{\theta^{2}}, V_{r}\right\}$ and, therefore,

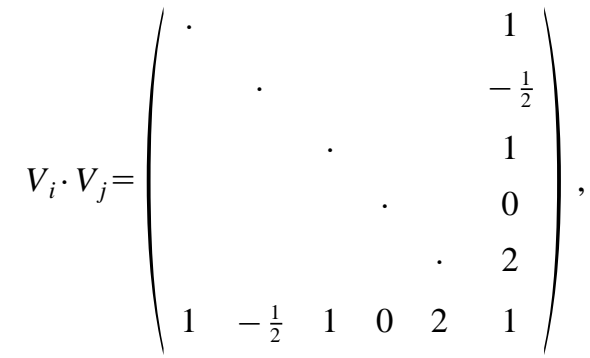

$$
\begin{aligned}
& k_{i j}=\left(\begin{array}{cccccc}
\cdot & & & & & k_{r 0} \\
& \cdot & & & & k_{r 1+\frac{1}{2}} \\
& & \cdot & & & k_{r 2} \\
& & & \cdot & & k_{r 3} \\
& & & & \cdot & k_{r \theta^{2}} \\
k_{r 0} & k_{r 1} & k_{r 2} & k_{r 3} & k_{r \theta^{2}} & k_{r 0}
\end{array}\right) .
\end{aligned}
$$

The gauge group is broken further by $V_{r}$ to $[\mathrm{SO}(10)]^{2} \times[\mathrm{U}(1)]^{2} \times[\mathrm{SO}(4)]^{4} \times \mathrm{U}(1) \times \mathrm{SO}(6) \times[\mathrm{U}(1)]^{2}$. The supersymmetry is reduced to $N=1$ due to the extra constraint:

$$
V_{r} \cdot N_{V_{1}}=\frac{1}{2}\left(N_{1}+N_{5}\right)=k_{r 1}+\frac{1}{2}-V_{r} \cdot V_{1}=k_{r 1} .
$$

The $V_{r}$ sector has vacuum energy $[0,0]$. The spectrumgenerating formula reads

$$
\begin{gathered}
\frac{1}{2} N_{1}+\frac{1}{2}\left(\widetilde{N}_{1}+\widetilde{N}_{3}+\cdots+\widetilde{N}_{9}\right)=k_{2 r}-\frac{1}{2} \quad(\bmod 1), \\
\frac{1}{2}\left(\widetilde{N}_{1}+\widetilde{N}_{3} \cdots+\widetilde{N}_{15}\right)=k_{\theta^{2} r} \quad(\bmod 1) \\
\frac{1}{2}\left(N_{1}+N_{5}\right)=k_{1 r} \quad(\bmod 1) \\
\frac{1}{2}\left(N_{10}+\widetilde{N}_{11}\right)=k_{3 r} \quad(\bmod 1) \\
\frac{1}{2}\left(N_{1}+N_{3}+N_{5}+N_{10}\right)=k_{\theta^{2} r}+k_{0 r}-\frac{1}{2} \quad(\bmod 1) .
\end{gathered}
$$

It contributes chiral fermions in the representation $(\mathbf{1 6}, \mathbf{1}, \mathbf{2}, \mathbf{1})+(\mathbf{1 6}, \mathbf{1}, \overline{\mathbf{2}}, \mathbf{1}) \quad$ or $\quad(\overline{\mathbf{1 6}}, \mathbf{1}, \mathbf{2}, \mathbf{1})+(\overline{\mathbf{1 6}}, \mathbf{1}, \overline{\mathbf{2}}, \mathbf{1}) \quad$ of $[\mathrm{SO}(10)]^{2} \times[\mathrm{SO}(4)]^{2}$ [the first two $\left.\mathrm{SO}(4)\right]$ depending on the space-time helicity. The $V_{r}+V_{\theta^{2}}$ sector differs from the $V_{r}$ sector by an interchange of the first 16 left-moving NS and $\mathrm{R}$ fermions and so contributes chiral fermions in the representation $(\mathbf{1}, \mathbf{1 6}, \mathbf{1}, \mathbf{2})+(\mathbf{1}, \mathbf{1 6}, \mathbf{1}, \overline{\mathbf{2}})$ or $(\mathbf{1}, \overline{\mathbf{1 6}}, \mathbf{1}, \mathbf{2})$ $+(\mathbf{1}, \overline{\mathbf{1 6}}, \mathbf{1}, \overline{\mathbf{2}})$. Altogether, there are eight families of chiral fermions in the $\mathbf{1 6}$ (or $\overline{\mathbf{1 6}}$ ) of $\mathrm{SO}(10)$.

The analysis of $Z_{r \theta}$ is similar and we shall be brief. With the generating vectors $\left\{V_{0}, \ldots, V_{3}, V_{\theta^{2}}, V_{r \theta}\right\}$, 


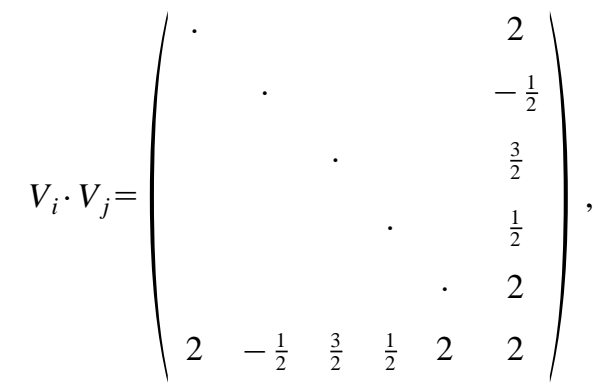



Again, gauge bosons only come from the $\mathbf{0}$ sector and with the addition of $V_{r \theta}$, the gauge group is broken to $[\mathrm{SO}(10)]^{2} \times[\mathrm{U}(1)]^{2} \times[\mathrm{SO}(4)]^{2} \times[\mathrm{U}(1)]^{2} \times \mathrm{SO}(4) \times[\mathrm{U}(1)]^{2}$.

The two gravitinos in the $V_{1}$ sector are either all projected out (if $k_{r \theta, 1} \neq k_{21}$ ) or remain untouched (if $k_{r \theta, 1} \neq k_{21}$ ).

We finally come to $Z_{\theta}$ which is generated by $\left\{V_{0}, \ldots, V_{3}, V_{\theta}\right\}$. Therefore,

$$
V_{i} \cdot V_{j}=\left(\begin{array}{ccccc}
\cdot & & & 0 \\
& \cdot & & -\frac{1}{2} \\
& & \cdot & 0 \\
& & & \cdot & \frac{1}{2} \\
0 & -\frac{1}{2} & 0 & \frac{1}{2} & 1
\end{array}\right),
$$

The gauge group is broken to $\mathrm{U}(10) \times \mathrm{U}(2) \times \mathrm{U}(4)$ $\times[\mathrm{U}(1)]^{2} \times \mathrm{SO}(4) \times[\mathrm{U}(1)]^{2}$. In contrast with $V_{r}, V_{\theta}$ breaks the supersymmetry to $N=1$ but does not introduce chiral fermions.

What can we say about the resultant $D_{4}$ orbifold simply by counting? If we choose $k_{r \theta, 1}=k_{21}$, there are two gravitinos in $Z_{r \theta}$ and the resultant model has $N=1$ supersymmetry. In addition, there are 68 gauge bosons and four families of chiral fermions in the $\mathbf{1 6}$ of $\mathrm{SO}(10)$.

A thorough examination of the spectrum (such as identification of the gauge group) requires a more careful treatment. There are 27 potentially massless sectors, 13 of which are projected out by the physically sensible projection. The model is supersymmetric and so it suffices to list only half of the remaining sectors (the others can be obtained by adding
$\left.V_{1}\right)$. The untwisted sectors include $\mathbf{0}, V_{2}+V_{\theta^{2}}$, and $V_{2}+V_{3}+V_{\theta^{2}}$ while the twisted sectors include $V_{r}$, $V_{r}+V_{\theta^{2}}, V_{\theta}$, and $3 V_{\theta}$.

The 68 gauge bosons all come from the $\mathbf{0}$ sector. In the basis such that $\theta$ is diagonal, the spectrum-generating formula gives

$$
\begin{gathered}
\frac{1}{2}\left(N_{1}+\cdots+N_{10}\right)+\frac{1}{2}\left(\widetilde{N}_{1}+\cdots+\widetilde{N}_{22}\right)=\frac{1}{2} \quad(\bmod 1), \\
\frac{1}{2}\left(N_{1}+N_{2}+N_{5}+N_{8}\right)=\frac{1}{2} \quad(\bmod 1), \\
\frac{1}{2}\left(N_{1}+N_{2}+N_{6}+N_{9}\right)+\frac{1}{2}\left(\widetilde{N}_{1}+\cdots+\widetilde{N}_{10}+\widetilde{N}_{21}+\widetilde{N}_{22}\right)=\frac{1}{2} \\
\quad(\bmod 1), \quad(4.34) \\
\frac{1}{2}\left(N_{9}+N_{10}\right)+\frac{1}{2}\left(\widetilde{N}_{11}+\widetilde{N}_{12}+\widetilde{N}_{18}+\cdots+\widetilde{N}_{21}\right)=0
\end{gathered}
$$

$(\bmod 1)$,

$$
\begin{gathered}
\frac{1}{2}\left(N_{1}+N_{3}+N_{6}+N_{8}\right)+\frac{1}{4}\left(\widetilde{N}_{1}-\widetilde{N}_{2}+\cdots+\widetilde{N}_{15}-\widetilde{N}_{16}\right) \\
+\frac{1}{2}\left(\widetilde{N}_{17}+\widetilde{N}_{18}+\widetilde{N}_{21}+\widetilde{N}_{22}\right)=\frac{1}{2} \quad(\bmod 1)
\end{gathered}
$$

The states that survive the projection are invariant under $\theta$. As mentioned before, the gauge bosons form the adjoint representation of $\mathrm{U}(10) \times \mathrm{U}(2) \times \mathrm{U}(4) \times[\mathrm{U}(1)]^{2} \times \mathrm{SO}(4) \times \mathrm{U}(1)^{2}$. Take the $\mathrm{U}(10)$ bosons for example. They are obtained by exciting $\widetilde{N}_{i}=\left(\delta_{i j}-\delta_{i k}\right)$ for $j, k=1,3, \ldots, 9$ or $2,4, \ldots, 10$ or by exciting $\widetilde{N}_{i}= \pm\left(\delta_{i j}+\delta_{i k}\right)$ for $j=1,3, \ldots, 9$ and $k=2,4, \ldots, 10$.

These states, however, are not invariant under the full non-Abelian group $D_{4}$. Under the action of $r, \widetilde{N}_{2 i-1}$ and $\widetilde{N}_{2 i}$ for $i=1, \ldots 8$, are interchanged. Nevertheless, we can obtain group-invariant states by forming linear combinations of $\theta$-invariant states. In other words, let $|\theta\rangle$ be an $\theta$-invariant state, then the combination $|\theta\rangle+r|\theta\rangle$ is invariant under $D_{4}$. It follows that the gauge bosons invariant under $D_{4}$ form the adjoint representation of $\mathrm{SO}(10)_{2}$ $\times \mathrm{U}(1) \times \mathrm{SO}(4)_{2} \times[\mathrm{U}(1)]^{2} \times \mathrm{SO}(4) \times[\mathrm{U}(1)]^{2}$. The rank of the gauge group is reduced to 14 . It can easily be verified that the $\mathrm{SO}(10)$ and the $\mathrm{SO}(4)$ are realized at level 2 by examining the operator product expansion (OPE) of their KacMoody currents. We may regard the $\mathrm{SO}(10)_{2}$ as the observable sector and the rest as the hidden sector. In what follows, we will only consider states that are not singlets under $\mathrm{SO}(10)$.

In addition to gauge bosons, the $\mathbf{0}$ sector also contributes scalars. For example, take $N_{i}= \pm \delta_{i 2}$ and $\widetilde{N}_{i}$ the same as that of the gauge bosons, we have two families of scalars in the 45 of $\mathrm{SO}(10)$. In addition, there are scalars in the $(\mathbf{1 0 , 4 )}$ of $\mathrm{SO}(10) \times \mathrm{SO}(4)$.

The analysis of the twisted sectors is similar except that group invariance requires mixing of states from conjugate sectors. To illustrate this, consider a physical state in the 
$V_{r}$ sector. It is invariant under $r$ and $\theta^{2}$ and we denote it as $\left|r, \theta^{2}\right\rangle$. The group-invariant combination is thus,

$$
\left|r, \theta^{2}\right\rangle+\theta\left|r, \theta^{2}\right\rangle .
$$

Here, $\theta\left|r, \theta^{2}\right\rangle$ is in the conjugate sector $V_{r}+V_{\theta^{2}}$ because $\theta r \theta^{3}=r \theta^{2}$. As a result, the conjugate sectors together contribute four families of chiral fermions in the $\mathbf{1 6}$ of $\mathrm{SO}(10)$.

The other sectors only contribute $\mathrm{SO}(10)$ singlets. To summarize, the resultant model has observable gauge group $\mathrm{SO}(10)$ realized at level 2, $N=1$ supersymmetry, four chiral families of $\mathrm{SO}(10)$, and massless scalars in the adjoint of $\mathrm{SO}(10)$

So far we have limited ourselves to only one-dimensional representations on the right movers. With the $[\mathrm{SU}(2)]^{6}$ form of the supercurrent,

$$
T_{F}=i \psi^{u} \partial X_{u}+i \sum_{\ell=1}^{6} \psi_{1}^{\ell} \psi_{2}^{\ell} \psi_{3}^{\ell},
$$

a general automorphism that respects world-sheet supersymmetry is a product of an inner automorphism for the individual $\mathrm{SU}(2)$ algebras and an outer automorphism that permutes the different $\mathrm{SU}(2)$. This allows a truly non-Abelian action on the right movers since, in general, two such automorphisms do not commute.

Consider two noncommuting automorphisms $\theta$ and $r$ of $[\mathrm{SU}(2)]^{2}$ defined by

$$
\begin{gathered}
\theta: \psi_{j}^{1} \rightarrow \psi_{j}^{2}, \\
\psi_{j}^{2} \rightarrow \psi_{j}^{1} \sigma_{j}, \\
r: \psi_{j}^{1} \rightarrow \psi_{j}^{2}, \\
\psi_{j}^{2} \rightarrow \psi_{j}^{1},
\end{gathered}
$$

where $\sigma_{1}=\sigma_{2}=-1, \sigma_{3}=1$. It is obvious that $\theta^{4}=r^{2}=1$ and $r \theta^{3}=\theta r$. Therefore, the group generated by $\theta$ and $r$ is $D_{4}$.

The eigenstates and the corresponding eigenvalues of $\theta$ are

$$
\begin{array}{cc}
\chi_{r}^{1}=\psi_{3}^{1}+\psi_{3}^{2}, & \theta=1 ; \\
\chi_{r}^{2}=-\psi_{3}^{1}+\psi_{3}^{2}, & \theta=-1 ; \\
\chi^{3}=i \psi_{1}^{1}-\psi_{1}^{2}, & \theta=-i ; \\
\chi^{4}=-i \psi_{2}^{1}-\psi_{2}^{2}, \quad \theta=i ;
\end{array}
$$

where $\chi$ with the subscript $r$ are real fermions. Similarly, for $r$,

$$
\begin{array}{cc}
\chi_{r}^{1}=\psi_{3}^{1}+\psi_{3}^{2}, & r=1 ; \\
\chi_{r}^{2}=-\psi_{3}^{1}+\psi_{3}^{2}, & r=-1 ; \\
\chi^{3}=\psi_{1}^{1}+\psi_{1}^{2}, & r=1 ; \\
\chi^{4}=-\psi_{2}^{1}+\psi_{2}^{2}, & r=-1 .
\end{array}
$$

To illustrate how to construct non-Abelian orbifolds with nontrivial SU(2) outer automorphisms, let us consider a symmetric $D_{4}$ orbifold of the model generated by $\left\{V_{0}, V_{1}\right\}$. The $D_{4}$ action is specified by the generating vectors:

$$
\begin{aligned}
V_{\theta}= & {\left[-\frac{1}{2}\left\{0_{r},\left(-\frac{1}{2}\right)_{r},-\frac{1}{4}, \frac{1}{4}\right\}^{2}\left(0-\frac{1}{2} 0\right) \mid\right.} \\
& \left.-\frac{1}{2}\left\{0_{r},\left(-\frac{1}{2}\right)_{r},-\frac{1}{4}, \frac{1}{4}\right\}^{2}\left(0-\frac{1}{2} 0\right) 0^{12}\right], \\
V_{r}= & {\left[-\frac{1}{2}\left\{0_{r},\left(-\frac{1}{2}\right)_{r}, 0,-\frac{1}{2}\right\}^{2}\left(0-\frac{1}{2} 0\right) \mid\right.} \\
& \left.-\frac{1}{2}\left\{0_{r},\left(-\frac{1}{2}\right)_{r}, 0,-\frac{1}{2}\right\}^{2}\left(0-\frac{1}{2} 0\right) 0^{12}\right] .
\end{aligned}
$$

In other words, two copies of $[\mathrm{SU}(2)]^{2}$ transform under $\theta$ and $r$ defined above. The remaining fermions are simply one-dimensional representations of $D_{4}$.

In order that the Abelian models are well defined, the original set of generating vectors must commute with the group action. It is easy to see that $V_{0}$ and $V_{1}$ commute with both $V_{\theta}$ and $V_{r}$. The non-Abelian rules impose constraints on the structure constants:

$$
\begin{gathered}
k_{\theta^{2} j}=0, \\
k_{r j}+k_{\theta j}=k_{r \theta, j}, \\
k_{r \theta^{2}}=k_{r \theta, \theta^{2}},
\end{gathered}
$$

where $j=0,1$. It follows immediately from $k_{\theta^{2} 1}=0$ that $Z_{\theta^{2}}$ remains $N=4$ supersymmetric. The gauge group is broken to $\mathrm{SO}(8) \times \mathrm{SO}(36)$. Both $Z_{r}$ and $Z_{\theta}$ have $N=2$ supersymmetry with gauge groups $\mathrm{SO}(4) \times \mathrm{SO}(4) \times \mathrm{SO}(6) \times \mathrm{SO}(30)$ and $\mathrm{SO}(4) \times \mathrm{SO}(4) \times \mathrm{SO}(36)$, respectively. $Z_{r \theta}$ has gauge group $\mathrm{U}(4) \times \mathrm{SO}(6) \times \mathrm{SO}(30)$. Depending on $k_{r \theta, 1}$, the $N=4$ supersymmetry in $Z_{r \theta}$ is unbroken $\left(k_{r \theta, 1}=0\right)$ or completely broken $\left(k_{r \theta, 1}=\frac{1}{2}\right)$. As usual, rank reduction and higher-level gauge group come with the non-abelian orbifold. The resultant $D_{4}$ orbifold has rank 20 gauge group $\mathrm{SO}(4)_{2} \times \mathrm{SO}(6) \times \mathrm{SO}(30)$ and $N=2$ (or 0$)$ supersymmetry.

Similar analyses can be carried out for non-Abelian groups generated by other automorphisms of $[\mathrm{SU}(2)]^{6}$. We may also start with a model generated by more basis vectors, provided that they commute with the automorphisms. A complete classification of the $[\mathrm{SU}(2)]^{6}$ automorphisms as well as the sets of mutually commuting basis vectors have been studied in Ref. [13].

\section{BOSONIC FORMULATION}

The above rules can be easily generalized to the general orbifold case. This generalization is easiest if we start with the recent rules for asymmetric orbifold [6], since they are brought to a form very similar to that for the free fermionic string construction. Because the generalization in this formulation is completely analogous to that for the free fermionic construction, we shall be brief.

Let us first review the Abelian orbifold case. Again, let us consider heterotic strings compactified to four space-time dimensions. In the light-cone gauge, which we adopt, we have the following world-sheet degrees of freedom: One complex boson $\phi^{0}$ (corresponding to two transverse space-time coordinates); three right-moving complex bosons $\phi_{R}^{\prime}, \ell=1,2,3$ 
(corresponding to six internal coordinates); four rightmoving complex fermions $\psi^{r}, r=0,1,2,3$ ( $\psi^{0}$ is the worldsheet superpartner of the right-moving component of $\phi^{0}$, whereas $\psi$ are the world-sheet superpartners of $\phi_{R}^{\ell}$, $\ell=1,2,3) ; 11$ left-moving complex bosons $\phi_{L}$, $\ell=4,5, \ldots, 14$ (corresponding to twenty-two internal coordinates). Before orbifolding, the corresponding string model has $N=4$ space-time supersymmetry and the internal momenta span an even self-dual Lorentzian lattice $\Gamma^{6,22}$.

It is convenient to organize the string states into sectors labeled by the monodromies of the string degrees of freedom. These monodromies can be combined into a single vector. For each complex boson we will specify either $T_{i}^{\ell}$ (twist), or $U_{i}^{\ell}$ (shift). To keep track of whether a given entry corresponds to a twist or to a shift, it is convenient to introduce auxiliary vectors

$$
W_{i}=\left[0\left(0 W_{i}^{1}\right)\left(0 W_{i}^{2}\right)\left(0 W_{i}^{2}\right) \| W_{i}^{4} \cdots W_{i}^{14}\right] .
$$

The double vertical lines separate the right and left movers. The entries $W_{i}^{\ell}$ are defined as follows: $W_{i}^{\ell}=\frac{1}{2}$ if $T_{i}^{\ell} \neq 0$; $W_{i}^{\ell}=0$, otherwise. For example,

$$
\begin{gathered}
V_{i}=\left[V_{i}^{0}\left(V_{i}^{1} T_{i}^{1}\right)\left(V_{i}^{2} T_{i}^{2}\right)\left(V_{i}^{3} T_{i}^{3}\right) \| U_{i}^{4} \cdots U_{i}^{13} T_{i}^{14}\right], \\
W_{i}=\left[0\left(0 \frac{1}{2}\right)^{3} \| 0^{10 \frac{1}{2}}\right],
\end{gathered}
$$

where $T_{i}^{1}, T_{i}^{2}, T_{i}^{3}$, and $T_{i}^{14}$ correspond to twists, $U_{i}^{4} \ldots$, $U_{i}^{13}$ correspond to shifts, and $V_{i}^{r} r=0,1,2,3$, specify the fermionic spin structures. Without loss of generality we can restrict the values of $V_{i}^{r}$ and $T_{i}^{\ell}$ as follows: $-\frac{1}{2} \leqslant V_{i}^{r}<\frac{1}{2}$; $0 \leqslant T_{i}^{\ell}<1$ [a complex boson (fermion) with boundary condition $T_{i}^{\ell}\left(V_{i}^{r}\right)=0$ or $\frac{1}{2}$ can be split into two real bosons (fermions)]. The shifts $U_{i}^{\ell}$ can be combined into a real $(6,22)$-dimensional Lorentzian vector $\vec{U}_{i}$ defined up to the identification $\vec{U}_{i} \sim \vec{U}_{i}+\vec{P}$, where $\vec{P}$ is an arbitrary vector of $\Gamma^{6,22}$.

The notation we have introduced proves convenient in describing the sectors of a given string model based on the orbifold group $G$. For $G$ to be a finite Abelian group, the element $g\left(V_{i}\right)$ must have a finite order $m_{i} \in \mathbf{N}$, i.e., $g^{m_{i}}\left(V_{i}\right)=1$. This implies that $V_{i}^{r}$ and $T_{i}^{\ell}$ must be rational numbers, and the shift vector $\vec{U}_{i}$ must be a rational multiple of a vector in $\Gamma^{6,22}$; that is, $m_{i} V_{i}^{r}, m_{i} T_{i}^{\ell} \in \mathbf{Z}$, and $m_{i} \vec{U}_{i}$ $\in \Gamma^{6,22}$. To describe all the elements of the group $G$, it is convenient to introduce the set of generating vectors $\left\{V_{i}\right\}$ such that $\overline{\alpha V}=\mathbf{0}$ if and only if $\alpha_{i} \equiv 0$. Here, $\mathbf{0}$ is the null vector: $\mathbf{0}=\left[0(00)^{3} \| 0^{11}\right]$. Also, $\alpha V \equiv \sum_{i} \alpha_{i} V_{i}$ [the summation is defined as $\left.\left(V_{i}+V_{j}\right)^{\ell}=V_{i}^{\ell}+V_{j}^{\ell}\right], \alpha_{i}$ being integers that take values from 0 to $m_{i}-1$. The overbar notation is defined as follows: $\overline{\alpha V} \equiv \alpha V-\Delta(\alpha)$, and the components of $\overline{\alpha V}$ satisfy $-\frac{1}{2} \leqslant \overline{\alpha V}^{r}<\frac{1}{2}, 0 \leqslant \overline{\alpha T}^{\ell}<1$; here, $\Delta^{r}(\alpha), \Delta^{\ell}(\alpha)$ $\in \mathbf{Z}$. So the elements of the group $G$ are in one-to-one correspondence with the vectors $\overline{\alpha V}$ and will be denoted by $g(\overline{\alpha V})$. It is precisely the Abelian nature of $G$ that allows this correspondence (by simply taking all possible linear combinations of the generating vectors $V_{i}$ ). So the sectors of the model are labeled by the vectors $\overline{\alpha V}$. To maintain worldsheet supersymmetry, each vector must satisfy the following supercurrent constraint

$$
V_{i}^{\ell}+T_{i}^{\ell}=V_{i}^{0} \equiv s_{i} \quad(\bmod 1), \quad \ell=1,2,3
$$

Here $s_{i}$ is the monodromy of the supercurrent $\bar{S}\left(\bar{z} e^{-2 \pi i}\right)=\exp \left(2 \pi i s_{i}\right) \bar{S}(\bar{z})$, which must satisfy $s_{i} \in \frac{1}{2} \mathbf{Z}$. Then, the sectors with $\overline{\alpha V}^{0}=0$ give rise to space-time bosons, while the sectors with $\overline{\alpha V}^{0}=-\frac{1}{2}$ give rise to spacetime fermions. This is the bosonic equivalence of the triplet constraint (2.2).

The rules for model building are quite similar to those for the free fermionic string construction. To obtain relatively simple rules for building asymmetric orbifold models, we will confine our attention to the orbifolds with twists whose orders are co-prime numbers. [The order of a twist generated by a vector $\overline{\alpha V}$ is defined as the smallest positive integer $t(\overline{\alpha V})$, such that $\forall \ell t(\overline{\alpha V}) \overline{\alpha T} \ell \in \mathbf{Z}$; note that $t(\overline{\alpha V})$ is a divisor of $m(\overline{\alpha V})$.] In this case, a given model can be viewed as being generated by a single twist $V^{*}$ of order $t^{*}=\Pi_{i} t_{i}$, such that $\overline{\alpha V}=\overline{\alpha^{*} V^{*}}$, where $\alpha^{*} / t^{*}=\Sigma_{i} \alpha_{i} / t_{i}(\bmod 1)$, and $\alpha^{*}$ takes values $0,1, \ldots, t^{*}-1$. We may work in the $V^{*}$ basis or the $\left\{V_{i}\right\}$ basis.

In a given sector $\overline{\alpha V}$, the right- and left-moving Hamiltonians are given by the corresponding sums of the Hamiltonians for individual string degrees of freedom. The Hilbert space in the $\overline{\alpha V}$ sector is given by the momentum states $\left|\vec{P}_{\alpha V}+\alpha \vec{U}, \mathbf{n}\right\rangle$, and also the states obtained from these states by acting with the fermion and boson creation operators (oscillator excitations). $\mathbf{n}$ is a collective notation for $n_{i}=0,1, \ldots, t_{i}-1$. In the untwisted sectors, that is, sectors $\overline{\alpha V}$ with $t(\overline{\alpha V})=1$, we have $\vec{P} \overline{\alpha V} \in \Gamma^{6,22}$. In the twisted sectors $\overline{\alpha V}$ with $t(\overline{\alpha V}) \neq 1$, we have $\vec{P}_{\overline{\alpha V}} \in \widetilde{I}(\overline{\alpha V})$, where $\widetilde{I}(\overline{\alpha V})$ is the lattice dual to the lattice $I(\overline{\alpha V})$, which in turn is the sublattice of $\Gamma^{6,22}$ invariant under the action of the twist part of the group element $g(\overline{\alpha V})$. The ground states $|\overrightarrow{0}, \mathbf{n}\rangle$ in the $\overline{\alpha V}$ sector appear with certain degeneracies $\xi(\overline{\alpha V}, \mathbf{n})$, which are non-negative integers. In the untwisted sectors $\xi(\overline{\alpha V}, \mathbf{n})=1$ if all $n_{i}=0$, and zero otherwise. In the twisted sectors, the situation is more involved. Here, the states with quantum numbers $\mathbf{n}$ appear with the multiplicity $\xi(\overline{\alpha V}, \mathbf{n})$, with fixed point phase $f_{i}(\overline{\alpha V}, \mathbf{n})$ given by

$$
f_{i}(\overline{\alpha V}, \mathbf{n})=n_{i} / t_{i}
$$

The requirements of modular invariance and physically sensible projection unambiguously fix the degeneracies $\xi(\overline{\alpha V}, \mathbf{n})$. Now, the sum of $\xi(\overline{\alpha V}, \mathbf{n})$ over all $\mathbf{n}$ is the number of fixed points $\xi(\overline{\alpha V})$ in the $\overline{\alpha V}$ sector [2]:

$$
\sum_{\mathbf{n}} \xi(\overline{\alpha V}, \mathbf{n})=\xi(\overline{\alpha V})=[M(\overline{\alpha V})]^{-1 / 2} \prod_{\ell}\left[2 \sin \left(\pi \overline{\alpha T} T^{\ell}\right)\right]
$$

where the product over $\ell$ does not include the terms with $\overline{\alpha T} \ell=0$, and $M(\overline{\alpha V})$ is the determinant of the metric of $I(\overline{\alpha V})$. We also have the constraints 


$$
\xi(\overline{\alpha V}) \geqslant \sum_{\mathbf{n}} \xi(\overline{\alpha V}, \mathbf{n}) \exp [-2 \pi i \beta f(\overline{\alpha V}, \mathbf{n})]
$$

where the sum must be a nonzero integer. These constraints essentially fix all the $\xi(\overline{\alpha V}, \mathbf{n})$.

Since the momentum in the twisted sectors belongs to a shifted $\widetilde{I}(\overline{\alpha V})$ lattice, the level-matching condition requires that this lattice must have a prime $N(\overline{\alpha V})$, where $N(\overline{\alpha V})$ is the smallest positive integer such that for all vectors $\vec{P}$ $\in \widetilde{I}(\overline{\alpha V}), N(\overline{\alpha V}) \vec{P}^{2} \in 2 \mathbf{Z}$; moreover, for the corresponding characters to have the correct modular transformation properties (i.e., so that they are permuted under both $S$ and $T$ transformations), either $N(\overline{\alpha V})=1$ [in which case $I(\overline{\alpha V})$ is an even self-dual lattice], or $N(\overline{\alpha V})=t(\overline{\alpha V})$ [in which case $I(\overline{\alpha V})$ is even but not self-dual].

Imposing modular invariance and physically sensible projections, the following constraints on a consistent model emerge:

$$
\begin{gathered}
k_{i j}+k_{j i}=V_{i} \cdot V_{j} \quad(\bmod 1) \quad i \neq j, \\
k_{i i}+k_{i 0}+s_{i}-\frac{1}{2} V_{i} \cdot V_{i}=0 \quad(\bmod 1), \\
\left(k_{i j}-W_{i} \cdot V_{j}\right) m_{j}=0 \quad(\bmod 1), \\
m_{i} V_{i} \cdot\left[W(\overline{\alpha V})-\sum_{j} \alpha_{j} W_{j}\right]=0 \quad(\bmod 1) .
\end{gathered}
$$

Here, $V_{i} \cdot V_{j}$ is generalized to

$$
V_{i} \cdot V_{j}=\vec{U}_{i} \cdot \vec{U}_{j}-\sum_{r} V_{i}^{r} V_{j}^{r}+\sum_{\ell \text { :right }} T_{i}^{\ell} T_{j}^{\ell}-\sum_{\ell: \text { left }} T_{i}^{\ell} T_{j}^{\ell} .
$$

The spectrum-generating formula reads:

$$
\begin{aligned}
V_{i} \cdot \mathcal{N}_{\overline{\alpha V}}+f_{i}(\overline{\alpha V}, \mathbf{n})+\frac{\alpha^{*} t^{*}}{2 t_{i}} \vec{Q}^{2}(\vec{P} \overline{\alpha V}) \\
\quad=\sum_{j} k_{i j} \alpha_{j}+s_{i}-V_{i} \cdot[\overline{\alpha V}-W(\overline{\alpha V})] \quad(\bmod 1) .
\end{aligned}
$$

Here, $\vec{Q}(\vec{P} \overline{\alpha V}) \in \widetilde{I}(\overline{\alpha V})$ is an arbitrary vector such that $\vec{P} \overline{\alpha V}-\alpha^{*} \vec{Q}(\vec{P} \overline{\alpha V}) \in I(\overline{\alpha V})$. For example, for $\alpha^{*}=1$, we can choose $\vec{P} \overline{\alpha V}=\vec{Q}(\vec{P} \overline{\alpha V})$.

$$
\begin{aligned}
V_{i} \cdot \mathcal{N}_{\overline{\alpha V}} \equiv & \vec{U}_{i} \cdot \vec{P} \overline{\alpha V}-\sum_{r} V_{i}^{r} N_{\overline{\alpha V}}^{r}+\sum_{\text {:right }} T_{i}^{\ell} J_{\overline{\alpha V}}^{\ell} \\
& -\sum_{\ell: \text { left }} T_{i}^{\ell} J_{\overline{\alpha V}}^{\ell},
\end{aligned}
$$

where $N_{\overline{\alpha V}}^{r}$ are the fermion number operators, and $J_{\overline{\alpha V}}^{\ell}$ the boson number operators. Both have integer eigenvalues.

The states that satisfy the spectrum-generating formula include both on- and off-shell states. The on-shell states must satisfy the additional constraint that the left- and rightmoving energies are equal. In the $\overline{\alpha V}$ sector they are given by

$$
\begin{aligned}
& E_{\overline{\alpha V}}^{L}=-1+\sum_{\ell: \text { left }}\left\{\frac{1}{2} \overline{\alpha T}^{\ell}\left(1-\overline{\alpha T} T^{\ell}\right)\right. \\
& \left.+\sum_{q=1}^{\infty}\left[\left(q+\overline{\alpha T^{\ell}}-1\right) n_{q}^{\ell}+\left(q-\overline{\alpha T} T^{\ell}\right) \bar{n}_{q}^{\ell}\right]\right\} \\
& +\sum_{q=1}^{\infty} q\left(n_{q}^{0}+\bar{n}_{q}^{0}\right)+\frac{1}{2}\left(\vec{P} \frac{L}{\alpha V}+\alpha \vec{U}^{L}\right)^{2}, \\
& E \frac{R}{\alpha V}=-\frac{1}{2}+\sum_{\ell \text { :right }}\left\{\frac{1}{2} \overline{\alpha T}^{\ell}\left(1-\overline{\alpha T^{\ell}}\right)\right. \\
& \left.+\sum_{q=1}^{\infty}\left[\left(q+\overline{\alpha T}^{\ell}-1\right) m_{q}^{\ell}+\left(q-\overline{\alpha T}^{\ell}\right) \bar{m}_{q}^{\ell}\right]\right\} \\
& +\sum_{q=1}^{\infty} q\left(m_{q}^{0}+\bar{m}_{q}^{0}\right)+\frac{1}{2}\left(\vec{P}^{R} \overline{\alpha V}+\alpha \vec{U}^{R}\right)^{2} \\
& +\sum_{r}\left\{\frac{1}{2}\left(\overline{\alpha V}^{r}\right)^{2}+\sum_{q=1}^{\infty}\left[\left(q+\overline{\alpha V}^{r}-\frac{1}{2}\right) k_{q}^{r}\right.\right. \\
& \left.\left.+\left(q-\overline{\alpha V^{r}}-\frac{1}{2}\right) \bar{k}_{q}^{r}\right]\right\} \text {. }
\end{aligned}
$$

Here, $n_{q}^{\prime}$ and $\overrightarrow{n_{q}}$ are occupation numbers for the left-moving bosons $\phi_{L}^{\ell}$ whereas $m_{q}^{\ell}$ and $\bar{m}_{q}^{\ell}$ are those for the rightmoving bosons $\phi_{R}^{\ell}$. These take non-negative integer values. $k_{q}^{r}$ and $\bar{k}_{q}^{r}$ are the occupation numbers for the right-moving fermions, and they take only two values: 0 and 1 . The occupation numbers are directly related to the boson and fermion number operators. For example, $N_{\overline{\alpha V}}^{r}=\sum_{q=1}^{\infty}\left(k_{q}^{r}-\bar{k}_{q}^{r}\right)$.

To obtain non-Abelian orbifold models, with the nonAbelian group, we consider the Abelian orbifolds, whose Abelian groups are subgroups of the non-Abelian group. We follow the same approach given in Sec. III to obtain the consistency constraints on the sets of $\left\{k_{i j}, V_{i}\right\}$. Since the derivation and the rules are identical, we shall not repeat that approach here.

\section{EXAMPLES}

The simplest example is the $\mathbf{Z}_{2}$ orbifold of a single boson. This well-known example can also be recast in this formalism and is given in Appendix. Here, we give two orbifold models. The first model has the permutation group $S_{3}$ as the non-Abelian point group. The second one is simply the three-family $\mathrm{SO}(10)_{3}$ grand unification model given in Ref. [8], recast in the non-Abelian orbifold language. Its isometry group is $T \times \mathbf{Z}_{2}$, where $T$ is the tetrahedral group.

(1) The $S_{3}$ model. Let us start with the following fourdimensional Narain model, with $\Gamma^{6,22}=\Gamma^{6,6} \otimes \Gamma^{16}$, where $\Gamma^{16}$ is the $\operatorname{spin}(32) / \mathbf{Z}_{2}$ lattice, and $\Gamma^{6,6}=\left(\Gamma^{1,1} \otimes \Gamma^{2,2}\right)^{2}$ where both $\Gamma^{1,1}$ and $\Gamma^{2,2}$ are specific even self-dual lattices: $\Gamma^{1,1}=\left\{\left(p_{R}|| p_{L}\right) \mid p_{R}=1 / \sqrt{2}(m-n), \quad p_{L}=1 / \sqrt{2}(m+n) ;\right.$ $m, n \in \mathbf{Z}\}$, i.e., an $\mathrm{SU}(2)$ lattice and $\Gamma^{2,2}$ 
$=\left\{\left(\vec{p}_{R}|| \vec{p}_{L}\right) \mid \vec{p}_{R, L}=(1 / 2 R) \vec{e}^{i} m_{i} \overline{+} R e_{i} n^{i}\right\}$ where $e_{i}$ and $\vec{e}^{i}$ are the SU(3) simple roots and their duals and $R$ is the compactification radius. This $N=4$ model (called $N 4$ ) has $\mathrm{SU}(2) \times \mathrm{SU}(2) \times \mathrm{U}(1)^{2} \times \mathrm{U}(1)^{2} \times \mathrm{SO}(32)$ gauge group.

Let us first consider the $\mathbf{Z}_{3}$ orbifold:

$$
\begin{gathered}
V_{0}=\left[-\frac{1}{2}\left(-\frac{1}{2} 0\right)_{r}^{2}\left(\frac{1}{2} 0\right)^{2}|| 0_{r}^{2} 0^{2} \mid 0_{r}^{16}\right], \\
V_{1}=\left[0(00)_{r}^{2}\left(-\frac{1}{3} \frac{1}{3}\right)\left(\frac{1}{3} \frac{2}{3}\right) \|\left(-\sqrt{\frac{2}{3}}\right)_{r}\left(\sqrt{\frac{2}{3}}\right)_{r} \frac{1}{3} \frac{2}{3} \mid\left(\frac{1}{3}\right)_{r}^{8}\left(\frac{2}{3}\right)_{r}^{2} 0_{r}^{6}\right],
\end{gathered}
$$

$$
W_{1}=\left[0(00)_{r}^{2}\left(0 \frac{1}{2}\right)^{2}|| 0_{r}^{2}\left(\frac{1}{2}\right)^{2} \mid 0_{r}^{16}\right],
$$

where the subscript $r$ labels real fermions and/or bosons. Since this is a symmetric $\mathbf{Z}_{3}$ twist, $V_{1} \cdot W_{1}=0$,

$$
V_{i} \cdot V_{j}=\left(\begin{array}{cc}
-1 & 0 \\
0 & 2
\end{array}\right), \quad k_{i j}=0 .
$$

Using the spectrum-generating formula (5.10), it is straightforward to work out the massless spectrum of this model. This orbifold yields an $N=2$ model (called $S 3$ ) which has the gauge symmetry $\mathrm{U}(1) \times \mathrm{U}(1) \times \mathrm{U}(10) \times \mathrm{SO}(12)$. Here, the $\mathrm{U}(1)$ factors come from $\Gamma^{1,1} \mathrm{~s}$.

Next, we consider a $\mathbf{Z}_{2}$ model. Let us consider the two bosons $\phi_{1}, \phi_{2}$ in $\Gamma^{2,2}$. Under this $\mathbf{Z}_{2}$ twist,

$$
\begin{gathered}
\phi_{2} \rightarrow-\phi_{2}, \\
\phi_{1} \rightarrow \phi_{1}+\phi_{2},
\end{gathered}
$$

i.e., it is a reflection that leaves $2 \phi_{1}+\phi_{2}$ invariant [note that since this reflection acts on both left- and right-moving momenta, it can be viewed as a rotation in a four-dimensional Euclidean lattice spanned by the vectors $\left(p_{R}|| p_{L}\right)$. In the example below, we could alternatively combine two reflections acting on the left-moving momenta into a $\mathbf{Z}_{2}$ rotation]. In fact, $\phi_{2}$ and $1 / \sqrt{5}\left(2 \phi_{1}+\phi_{2}\right)$ form the diagonal basis of this $\mathbf{Z}_{2}$ twist. Using this basis for both $\Gamma^{2,2}$, the $\mathbf{Z}_{2}$ orbifold is given by

$$
\begin{gathered}
V_{0}^{\prime}=\left[-\frac{1}{2}\left(-\frac{1}{2} 0\right)_{r}^{6}|| 0_{r}^{6} \mid 0_{r}^{16}\right], \\
V_{1}^{\prime}=\left\{-\frac{1}{2}\left(-\frac{1}{2} \frac{1}{2}\right)_{r}^{2}\left[\left(-\frac{1}{2} \frac{1}{2}\right)_{r}(00)_{r}\right]^{2}||\left(\frac{1}{2}\right)_{r}\left(\frac{1}{2} 0\right)_{r}^{2} \mid\left(-\frac{1}{2}\right)_{r}^{12} 0_{r}^{4}\right\},
\end{gathered}
$$

$$
W_{1}^{\prime}=\left\{0\left(0 \frac{1}{2}\right)_{r}^{2}\left[\left(0 \frac{1}{2}\right)_{r}(00)_{r}\right]^{2} \|\left(\frac{1}{2}\right)^{2}\left(\frac{1}{2} 0\right)_{r}^{2} \mid\left(\frac{1}{2}\right)_{r}^{12} 0_{r}^{4}\right\} .
$$

Here, $V_{1}^{\prime} \cdot W_{1}^{\prime}=3 / 2$, and

$$
V_{i}^{\prime} \cdot V_{j}^{\prime}=\left(\begin{array}{cc}
-1 & -\frac{1}{2} \\
-\frac{1}{2} & 1
\end{array}\right), \quad k_{i j}^{\prime}=\left(\begin{array}{cc}
0 & k_{10}^{\prime}+\frac{1}{2} \\
k_{10}^{\prime} & k_{10}^{\prime}
\end{array}\right) .
$$

Here, the two $\Gamma^{1,1}$ as well as $\Gamma^{16}$ are twisted. This $\mathbf{Z}_{2}$ orbifold yields an $N=2$ model (called $S 2$ ) with gauge symmetry $\mathrm{U}(1)^{4} \times \mathrm{SO}(12) \times \mathrm{SO}(20)$.

A comment on our choice of $\Gamma^{2,2}$ is necessary here. The invariant sublattice $I$ of $\Gamma^{2,2}$ under the $\mathbf{Z}_{3}$ twist consists of the origin $(\overrightarrow{0} \| \overrightarrow{0})$ only. On the other hand, the invariant sublattice $I^{\prime}$ of $\Gamma^{2,2}$ under the $\mathbf{Z}_{2}$ twist is nontrivial:

$$
I^{\prime}=\left\{\frac{m}{2 R}\left(\widetilde{e} \| \widetilde{e}^{-1}\right)+3 R n\left(-\widetilde{e}^{-1} \| \widetilde{e}^{-1}\right) ; m, n \in \mathbf{Z}\right\},
$$

where $\tilde{e}^{-1}$ is the dual of $e_{1}$. Here, $I^{\prime}$ is even but not self-dual. It is easy to find its dual $\widetilde{I}^{\prime}$,

$$
\widetilde{I^{\prime}}=\left\{\frac{m}{4 R}\left(\widetilde{e}^{-1} \| \widetilde{e}^{-1}\right)+\frac{3}{2} R n\left(-\widetilde{e}^{-1} \| \widetilde{e}^{-1}\right) ; m, n \in \mathbf{Z}\right\} .
$$

Recall $N_{I^{\prime}}$, where $N_{I^{\prime}} \vec{P}^{2} \in 2 \mathbf{Z}$ for $\vec{P} \in \widetilde{I^{\prime}}$. In this case, $N_{I^{\prime}}=t_{1}^{\prime}=2$, which is consistent. If we turn on appropriate antisymmetric background field and choose $R=2$ to get an enhanced SU(3) gauge symmetry, we will find that the corresponding $N_{I^{\prime}}=4$, which is unacceptable according to our rules. In fact, we must have zero antisymmetric background field. As a consequence, we have only $\mathrm{U}(1)^{2}$ gauge symmetry from each $\Gamma^{2,2}$.

Notice that the $\mathbf{Z}_{3}$ twist and the $\mathbf{Z}_{2}$ twist on $\Gamma^{2,2}$ do not commute; in fact, they generate the permutation group $S_{3}$ as the point group. So, the final orbifold involves a symmetric $S_{3}$ and an asymmetric $\mathbf{Z}_{2}$ twists. Since the $\mathbf{Z}_{3}$ shifts and the $\mathbf{Z}_{2}$ twist on part of $\Gamma^{16}$ do not commute, they generate the isometry group $D_{3}$, which is the same as $S_{3}$. The group $S_{3}$ has two generators $\theta$ and $r$ satisfying $\theta^{3}=r^{2}=1$ and $r \theta^{2}=\theta r$. It is divided into three conjugacy classes: $C_{1}=\{1\}, C_{\theta}=\left\{\theta, \theta^{2}\right\}$, and $C_{r}=\left\{r, r \theta, r \theta^{2}\right\}$. The stabilizer groups are given by $N_{1}=S_{3}, N_{\theta}=\left\{1, \theta, \theta^{2}\right\}$ and $N_{r}=\{1, r\}$. It is straightforward to check that the non-abelian constraints given in Sec. III are easily satisfied. The resulting orbifold has the partition function

$$
Z=\frac{1}{2} Z_{S 3}+Z_{S 2}-\frac{1}{2} Z_{N 4} .
$$

It is not difficult to work out the final massless spectrum of this $N=1$ supersymmetric model. The gauge symmetry, coming only from $\Gamma^{16}$, is $\mathrm{U}(1) \times \mathrm{SO}(10)_{2} \times \mathrm{SO}(10)_{1}$, where the subscripts indicate the levels of the current algebra $\mathrm{SO}(10)$.

(2) The three-family $\mathrm{SO}(10)_{3}$ model [8]. We start with an $N=4$ supersymmetric Narain model, which was referred to as $N 0$, with the lattice $\Gamma^{6,22}=\Gamma^{2,2} \otimes \Gamma^{4,4} \otimes \Gamma^{16}$. Here, $\Gamma^{2,2}=\left\{\left(p_{R} \| p_{L}\right)\right\}$ is an even self-dual Lorentzian lattice with $p_{R}, p_{L} \in \widetilde{\Gamma}^{2}\left[\mathrm{SU}(3)\right.$ weight lattice], $p_{L}-p_{R} \in \Gamma^{2}[\mathrm{SU}(3)$ root lattice]. Similarly, $\Gamma^{4,4}=\left\{\left(P_{R} \| P_{L}\right)\right\}$ is an even selfdual Lorentzian lattice with $P_{R}, P_{L} \in \widetilde{\Gamma}^{4}[\mathrm{SO}(8)$ weight lattice], $P_{L}-P_{R} \in \Gamma^{4} \quad\left[\mathrm{SO}(8)\right.$ root lattice]. $\Gamma^{16}$ is the $\operatorname{spin}(32) / \mathbf{Z}_{2}$ lattice. This model has $\mathrm{SU}(3) \times \mathrm{SO}(8) \times \mathrm{SO}(32)$ gauge group.

Next, consider the model generated by the vectors

$$
\begin{gathered}
V_{0}=\left[-\frac{1}{2}\left(-\frac{1}{2} 0\right)^{3} \| 0^{3} \mid 0_{r}^{16}\right], \\
V_{1}=\left[0\left(0 \frac{1}{2} e_{1}\right)\left(0 a_{1}\right)\left(0 b_{1}\right) \| 0^{3} \mid\left(\frac{1}{2}\right)_{r}^{5} 0_{r}^{5} 0_{r}^{5}\left(-\frac{1}{2}\right)_{r}\right], \\
V_{2}=\left[0\left(0 \frac{1}{2} e_{2}\right)\left(0 a_{2}\right)\left(0 b_{2}\right) \| 0^{3} \mid 0_{r}^{5}\left(\frac{1}{2}\right)_{r}^{5} 0_{r}^{5}\left(-\frac{1}{2}\right)_{r}\right] .
\end{gathered}
$$

Here, $e_{1}$ and $e_{2}$ are the simple roots of $\mathrm{SU}(3)$ $\left(e_{1} \cdot e_{1}=e_{2} \cdot e_{2}=-2 e_{1} \cdot e_{2}=2\right)$, whereas the fourdimensional real vectors $\boldsymbol{s}=\left(a_{1}, b_{1}\right)$ and $\boldsymbol{c}=\left(a_{2}, b_{2}\right)$ are, re- 
spectively, the spinor and conjugate weight vectors of $\mathrm{SO}(8)$. The generating vectors $V_{1}$ and $V_{2}$ are order-2 shifts $\left(m_{1}=m_{2}=2\right)$. They play the role of Wilson lines. The auxiliary vectors $W(\overline{\alpha V})$ are, therefore, null as there is no twisting of the lattice.

The matrix of the dot products $V_{i} \cdot V_{j}$ reads

$$
\begin{gathered}
V_{i} \cdot V_{j}=\left(\begin{array}{ccc}
-1 & 0 & 0 \\
0 & 0 & 1 \\
0 & 1 & 0
\end{array}\right), \\
k_{i j}=0 .
\end{gathered}
$$

The resulting model, which was referred to as $N 1$, is a Narain model with $N=4$ space-time supersymmetry and the gauge group $\mathrm{SU}(3) \times \mathrm{SO}(8) \times \mathrm{SO}(10)^{3} \times \mathrm{SO}(2)$. The details of this construction can be found in Refs. $[6,8]$.

Now, we consider the asymmetric orbifold model generated by the vectors

$$
\begin{gathered}
V_{0}=\left[-\frac{1}{2}\left(-\frac{1}{2} 0\right)^{3}|| 0^{3} \mid 0^{5} 0_{r}^{5} 0_{r}\right], \\
V_{1}^{\prime}=\left[0\left(-\frac{1}{3} \frac{1}{3}\right)^{3}|| 0\left(\frac{1}{3}\right)^{2} \mid\left(\frac{1}{3}\right)^{5} 0_{r}^{5}\left(\frac{2}{3}\right)_{r}\right], \\
W_{1}^{\prime}=\left[0\left(0 \frac{1}{2}\right)^{3}|| 0\left(\frac{1}{2}\right)^{2} \mid\left(\frac{1}{2}\right)^{5} 0_{r}^{5} 0_{r}\right] .
\end{gathered}
$$

The matrix of the dot products $V_{i}^{\prime} \cdot V_{j}^{\prime}$ reads

$$
V_{i}^{\prime} \cdot V_{j}^{\prime}=\left(\begin{array}{ll}
-1 & -\frac{1}{2} \\
-\frac{1}{2} & -\frac{1}{3}
\end{array}\right) .
$$

The structure constants $k_{i j}$ are then given by

$$
k_{i j}=\left(\begin{array}{cc}
0 & 0 \\
\frac{1}{2} & \frac{1}{3}
\end{array}\right) .
$$

Suppose, we start from the $N 1$ model, with the vectors (6.12) acting on it. This is the Abelian orbifold construction. Here, the right- and left-moving bosons corresponding to the $\mathrm{SU}(3) \times \mathrm{SO}(8)$ subgroup are complexified, whereas the single boson corresponding to the $\mathrm{SO}(2)$ subgroup is real, so are the five bosons $\varphi^{I} \equiv 1 / \sqrt{3}\left(\phi_{1}^{I}+\phi_{2}^{I}+\phi_{3}^{I}\right)$; the other ten real bosons are complexified via linear combinations $\Phi^{I} \equiv 1 / \sqrt{3}\left(\phi_{1}^{I}+\omega \phi_{2}^{I}+\omega^{2} \phi_{3}^{I}\right)$ and $\left(\Phi^{I}\right)^{\dagger} \equiv 1 / \sqrt{3}\left(\phi_{1}^{I}+\omega^{2} \phi_{2}^{I}\right.$ $+\omega \phi_{3}^{I}$ ), where $\omega=\exp \left(2 \pi i / 3\right.$ ) (the real bosons $\phi_{p}^{I}$ $I=1, \ldots, 5$, correspond to the $p$ th $\mathrm{SO}(10)$ subgroup, $p=1,2,3)$. Thus, the orbifold group element described by the $V_{1}$ vector permutes the real bosons corresponding to the three $\mathbf{S O}(10)$ 's. (This action is equivalent to a $\mathbf{Z}_{3}$ outer automorphism.) The resulting model (called $A 1$ ) is simply the $\mathrm{SO}(10)$ grand unification, with gauge symmetry $\mathrm{SU}(3)_{1} \times \mathrm{SU}(3)_{3} \times \mathrm{SO}(10)_{3} \times \mathrm{U}(1)$ and nine chiral $\mathrm{SO}(10)$ families. The details can be found in Refs. [6,8].

Notice that the world-sheet basis that is diagonal under the action of either of the Wilson lines (VI) is different from the basis that is diagonal under the $\mathbf{Z}_{3}$ twist. This implies that the $\mathbf{Z}_{3}$ twist action does not commute with the Wilson line action. So, if we want to start from the original $N 0$ model to reach the $A 1$ model, we must perform a non-Abelian orbi- fold, i.e., an isometry $\bar{P}$ composed of the Wilson lines (VI) and the $\mathbf{Z}_{3}$ twist plus shifts Eq. (6.12). Since the Wilson lines correspond to a $\mathbf{Z}_{2} \otimes \mathbf{Z}_{2}$ (shift) action, we see that $\bar{P}=T$, the tetrahedral group.

Let the resulting model coming from the above $\mathbf{Z}_{3}$ orbifold [i.e., $V_{0}, V_{1}^{\prime}$ of Eq. (6.12)] on the original $N 0$ model be referred to as the $B 1$ model. Again, it is easy to work out the massless spectrum of the $B 1$ model. It has $N=1$ supersymmetry and gauge symmetry $\mathrm{SU}(3)_{1} \times \mathrm{SU}(3)_{3} \times \mathrm{SU}(11)_{1} \times \mathrm{SO}(10)_{1} \times \mathrm{U}(1)$. Besides the usual gravity and gauge supermultiplets, this $B 1$ model also has, in its massless part of the spectrum, three copies of $(\mathbf{1}, \mathbf{1 0}, \mathbf{1}, \mathbf{1})(0)_{L}, \quad(\mathbf{1}, \mathbf{1}, \mathbf{1}, \mathbf{4 5})(0), \quad(\mathbf{1}, \overline{\mathbf{3}}, \mathbf{1}, \mathbf{1 6})(-1)_{L}$, $(\mathbf{1}, \overline{\mathbf{3}}, \mathbf{1}, \mathbf{1 0})(+2)_{L}$, and $(\mathbf{1}, \overline{\mathbf{3}}, \mathbf{1}, \mathbf{1})(-4)_{L}$. We see that all the massless states are singlets of $\mathrm{SU}(3)_{1} \times \mathrm{SU}(11)_{1}$. Note that the $\mathrm{SU}(3)_{3}$ chiral anomaly of the chiral field $(\mathbf{1}, \mathbf{1 0}, \mathbf{1}, \mathbf{1})(0)_{L}$ is canceled by those of the other fields, as a $\mathbf{1 0}_{L}$ of SU(3) has 27 times the anomaly contribution of a $\mathbf{3}_{L}$. The model is also $\mathrm{U}(1)$ anomaly-free due to the underlying $\mathrm{E}_{6}$ structure of the $\mathrm{SO}(10) \times \mathrm{U}(1)$ matter fields as can be seen from the branching $\mathbf{2 7}=\mathbf{1 6}(-1)+\mathbf{1 0}(+2)$ $+\mathbf{1}(-4)$ under $\mathrm{E}_{6} \supset \mathrm{SO}(10) \times \mathrm{U}(1)$.

Now, the $A 1$ model may be reached as the non-Abelian orbifold of the $N 0$ model, whose partition function is a linear combination of those of the $N 0$, the $N 1$, and the $B 1$ models. It is easy to see that the non-Abelian rules given in Sec. III are satisfied. The tetrahedral group $T$ has 12 elements generated by $r$ and $\theta$ satisfying $r^{2}=\theta^{3}$ $=(r \theta)^{3}=1$. There are four conjugacy classes: $C_{1}=\{1\}$, $C_{r}=\left\{r, r^{\prime}, r r^{\prime}\right\}, \quad C_{\theta}=\left\{\theta, r \theta, r^{\prime} \theta, r r^{\prime} \theta\right\}, \quad$ and $\quad C_{\theta^{2}}$ $=\left\{\theta^{2},(r \theta)^{2},\left(r^{\prime} \theta\right)^{2},\left(r r^{\prime} \theta\right)^{2}\right\}$ where $r^{\prime}=\theta r \theta^{-1}$. The stabilizer groups are given by $N_{1}=T, N_{r}=\left\{1, r, r^{\prime}\right\}$, and $N_{\theta}=\left\{1, \theta, \theta^{2}\right\}$. So the partition function of the $A 1$ model is given by

$$
Z_{A 1}=Z_{B 1}+\frac{1}{3} Z_{N 1}-\frac{1}{3} Z_{N 0}
$$

As mentioned above, the $A 1$ model has gauge symmetry $\mathrm{SU}(3)_{1} \times \mathrm{SU}(3)_{3} \times \mathrm{SO}(10)_{3} \times \mathrm{U}(1)$. In addition to the usual gravity and gauge supermultiplets, the massless spectrum of the resulting $A 1$ model has three copies of $(\mathbf{1}, \mathbf{1 0}, \mathbf{1})(0)_{L}$, $(\mathbf{1}, \mathbf{1}, \mathbf{4 5})(0), \quad(\mathbf{1}, \overline{\mathbf{3}}, \mathbf{1 6})(-1)_{L}, \quad(\mathbf{1}, \overline{\mathbf{3}}, \mathbf{1 0})(+2)_{L}, \quad$ and $(\mathbf{1}, \overline{\mathbf{3}}, \mathbf{1})(-4)_{L}$. Comparing the $A 1$ and the $B 1$ models, it seems that the Wilson lines (VI) remove the SU(11) gauge bosons in the $B 1$ model while raising the $\mathrm{SO}(10)$ current algebra level from 1 to 3 . This happens because the $\mathbf{Z}_{3}$ twist on the $\mathrm{SO}(32)$ in the $N 0$ model is an inner automorphism. So, this $\mathbf{Z}_{3}$ twist is equivalent to some $\mathbf{Z}_{3}$ shifts, which cannot change the current algebra level or cut the rank of the gauge group. On the other hand, the same $\mathbf{Z}_{3}$ twist on the SO $(10)^{3}$ in the $N 1$ model is an outer automorphism, which converts $\mathrm{SO}(10)^{3}$ to $\mathrm{SO}(10)_{3}$.

In the above example, one may prefer the two-step Abelian orbifold approach over the non-Abelian orbifold approach, since starting from the $N 1$ model is almost as easy as starting from the $N 0$ model. However, in the case where the point group is non-Abelian, such as the $S_{3}$ model discussed earlier, we do not see any obvious alternative. 
To reach the final three-family $\mathrm{SO}(10)$ model, one must reduce the number of chiral families from 9 to 3 by further orbifolding the $A 1$ model by a $\mathbf{Z}_{2}$ twist. This $\mathbf{Z}_{2}$ orbifold is generated by the following vectors:

$$
\begin{gathered}
V_{0}=\left[-\frac{1}{2}\left(-\frac{1}{2} 0\right)^{3}|| 0^{3} \mid 0^{5} 0_{r}^{5} 0_{r}\right], \\
V_{2}^{\prime}=\left[0(00)\left(-\frac{1}{2} \frac{1}{2}\right)^{2} \|\left(\frac{1}{2} e_{1}\right)\left(\frac{1}{2}\right)^{2} \mid 0^{5} 0_{r}^{5} 0_{r}\right], \\
W_{2}^{\prime}=\left[0(00)\left(0 \frac{1}{2}\right)^{2}|| 0\left(\frac{1}{2}\right)^{2} \mid 0^{5} 0_{r}^{5} 0_{r}\right] .
\end{gathered}
$$

The final model is the three-family $\mathrm{SO}(10)$ grand unified model presented in Ref. [8], with gauge symmetry $\mathrm{SU}(2)_{1} \times \mathrm{SU}(2)_{3} \times \mathrm{SO}(10)_{3} \times \mathrm{U}(1)^{3}$. Now, this $\mathbf{Z}_{2}$ twist commutes with the $\mathbf{Z}_{3}$ twist Eq. (6.12) and the Wilson lines Eq. (6.9). So, starting from the $N 0$ model, the orbifold group is $T \times \mathbf{Z}_{2}$.

There are alternative routes to the same final three-family $\mathrm{SO}$ (10) model. We may start with the $\mathbf{Z}_{2}$ orbifold (6.16) of the $N 0$ model, which we refer to as the $N 2$ model. If we now orbifold this $N 2$ model by the tetrahedral group $T$, the partition function of the final model is given by

$$
Z_{F}=Z_{A 3}+\frac{1}{3} Z_{N 3}-\frac{1}{3} Z_{N 2} \text {, }
$$

where $N 3$ is the $\mathbf{Z}_{2} \times \mathbf{Z}_{2}$ orbifold (i.e., $\left\{V_{0}, V_{1}, V_{2}\right\}$ ) of the $N 2$ model, and $A 3$ is the $\mathbf{Z}_{3}$ orbifold (B3) of the $N 2$ model. The final model is again the same three-family $\mathrm{SO}(10)$ grand unified model.

\section{ACKNOWLEDGMENTS}

This work was supported in part by the National Science Foundation.

\section{APPENDIX: THE $Z_{2}$ ORBIFOLD OF A SINGLE BOSON}

The $\mathbf{Z}_{2}$ orbifold of a single boson compactified at arbitrary radius is well understood. In this case, the orbifold involves a non-Abelian isometry group. It is, therefore, a good example to illustrate how the rules for constructing free fermionic string models and general Abelian orbifolds are generalized to include the construction of non-Abelian orbifolds. In particular, it will help clarify our notation. This example also illustrates the following point: how the same model $\Omega$ can be reached via an Abelian or a non-Abelian orbifold depending on the model $\Omega^{\prime}$ that we start with.

In the bosonic formulation, one can start with a torus $\Gamma$ of any desired radius and identify points on $\Gamma$ under an Abelian point group $P=\mathbf{Z}_{2}$. We can also start with a torus $\bar{\Gamma}$ of fixed radius 1 and obtain the same model by the combined action of (1) changing the radius, and (2) identifying points on the new torus $\Gamma$ under $P=\mathbf{Z}_{2}$. These two operations do not commute and generate the non-Abelian isometry group $\bar{P}$.

To be explicit, let us recall that $\bar{P}$ is a subgroup of the space group $S$ of rotations and translations. An element of $\bar{P}$, therefore, takes $X \rightarrow \theta X+v$ and is denoted by $(\theta, v)$. The multiplication law for elements of $\bar{P}$ is given by $(\theta, v)(\omega, u)=(\theta \omega, v+\theta u)$.

As an example, let us construct the $\mathbf{Z}_{2}$ orbifold at radius
$1 / n$ (for simplicity, we take $n$ to be odd). Starting from a torus of radius 1 , we can change the radius to $1 / n$ by modding out the lattice by a $\mathbf{Z}_{n}$ shift generated by $p=(1,1 / n)$. On the other hand, modding out the torus by a twist, $q=(-1,0)$, results in a $\mathbf{Z}_{2}$ orbifold. It is easy to verify that $p^{n}=q^{2}=1$ and $p q=q p^{n-1}$. Therefore, the group generated by $p$ and $q$ is $D_{n}$ which is divided into $\frac{1}{2}(n+3)$ conjugacy classes: $\quad C_{p^{m}}=\left\{p^{m}, p^{-m}\right\} \quad$ for $m=0, \ldots, \frac{1}{2}(n-1)$, and $C_{q}=\left\{q, p q, \cdots p^{n-1} q\right\}$. The stabilizer groups are $N_{p^{m}}=\left\{1, p, \ldots, p^{n-1}\right\} \quad$ and $\quad N_{p^{m} q}=\left\{1, p^{m} q\right\} \quad$ for $m=0, \ldots, n-1$. Therefore, the full partition function can be expressed as a sum of the three Abelian orbifolds:

$$
\begin{aligned}
Z & =\frac{1}{2} Z_{p}+\frac{1}{n} \sum_{m=0}^{n-1} Z_{q}^{m}-\frac{1}{2} Z_{1} \\
& =\frac{1}{2} Z_{p}+Z_{q}-\frac{1}{2} Z_{1},
\end{aligned}
$$

where $Z_{p}$ is the partition function of a single boson at radius $1 / n$, each $Z_{q}^{m}$ is equal to $Z_{q}$, a $\mathbf{Z}_{2}$ orbifold partition function at radius 1 , and $Z_{1}$ is the original partition function of a single boson at radius 1 , i.e., that of the starting model $\Omega^{\prime}$.

We now turn to the free fermionic construction. Consistency requires the presence of $V_{0}$. In the case of one complex world-sheet fermion, we always start from the model $\Omega^{\prime}$, which is equivalent to a single boson at radius 1 . Its partition function $Z_{1}$ is generated by a single vector

$$
V_{0}=\left(-\frac{1}{2} \mid-\frac{1}{2}\right) \text {. }
$$

It is easy to change the radius to $1 / n$ by introducing an additional basis vector $V_{p}$. The model $Z_{p}$ generated by

$$
\begin{aligned}
& V_{0}=\left(\frac{1}{2} \mid-\frac{1}{2}\right), \\
& V_{p}=\left(\frac{1}{n} \mid \frac{1}{n}\right),
\end{aligned}
$$

corresponds to a single boson at radius $1 / n$. We can also construct a $\mathbf{Z}_{2}$ orbifold by assigning different boundary conditions to the two real world-sheet fermions. The model $Z_{q}$ generated by

$$
\begin{gathered}
V_{0}=\left[\left(-\frac{1}{2}\right)^{2} \mid\left(-\frac{1}{2}\right)^{2}\right]_{r}, \\
V_{q}=\left(-\frac{1}{2} 0 \mid-\frac{1}{2} 0\right)_{r},
\end{gathered}
$$

is a $\mathbf{Z}_{2}$ orbifold at radius 1 . However, the vectors $V_{p}$ and $V_{q}$ are not compatible because $V_{p}$ must be in the complex fermion basis while $V_{q}$ is in the real fermion basis. So, the rules given in Refs. [3,9] cannot give the $\mathbf{Z}_{2}$ orbifold at radius $1 / n$. To construct this model, we must use the nonAbelian orbifold rules given in Sec. III. From Eq. (3.6), we see that $k_{i j}^{q, m}$ are the same for all $m$. Equation (3.7) implies that $k_{01}^{p}=k_{10}^{p}=k_{11}^{p}=0$ which are automatically satisfied. There is no new constraint from Eqs. (3.8) and (3.9). Therefore, $V_{p}$ and $V_{q}$ define a consistent model, and the resulting $Z$ is given by Eq. (A1).

By now, it is almost trivial to write this in the bosonic formulation. Let us start with the single boson at radius 1 
(i.e., model $Z_{1}$ ). In the real boson notation, the $Z_{p}$ model is given by the set of vectors acting on the $Z_{1}$ model,

$$
\begin{gathered}
V_{0}=(0 \| 0)_{r}, \\
V_{p}=\left(\frac{1}{n} \| \frac{1}{n}\right)_{r}, \\
W_{p}=(0 \| 0)_{r},
\end{gathered}
$$

and the $Z_{q}$ model is given the set of vectors acting on the $Z_{1}$ model,

$$
\begin{aligned}
V_{0} & =(0 \| 0)_{r}, \\
V_{q} & =\left(\frac{1}{2} \| \frac{1}{2}\right)_{r}, \\
W_{q} & =\left(\frac{1}{2} \| \frac{1}{2}\right)_{r} .
\end{aligned}
$$

Since this is a symmetric orbifold, the Abelian orbifold rules are trivial to apply, and we may choose $k_{i j}=0$. Here, the shift $V_{p}$ and the twist $V_{q}$ are the two generators of $D_{n}$, the isometry group $\bar{P}$. There is no simultaneously diagonalizable basis for $V_{p}$ and $V_{q}$ because the two operations do not commute. In the same fashion, we may apply our non-Abelian orbifold rules in Sec. III to deduce the consistency constraints on this $D_{n}$ orbifold. From Eq. (3.6), we again infer that $k_{i j}^{q, m}$ are the same for all $m$. Equation (3.7) implies that $k_{01}^{p}=k_{10}^{p}=k_{11}^{p}=0$ which are also automatically satisfied. There is no new constraints from Eqs. (3.8) and (3.9). Therefore, $V_{p}$ and $V_{q}$ define a consistent model provided that we interpret the $\mathbf{Z}_{n}$ shift $\left(V_{p}\right)$ and the $\mathbf{Z}_{2}$ twist $\left(V_{q}\right)$ as generators of the non-Abelian group $D_{n}$. The resulting $Z$ is again given by Eq. (A1).
[1] L. Dixon, J. Harvey, C. Vafa and E. Witten, Nucl. Phys. B261, 678 (1985); B274, 285 (1986).

[2] K. S. Narain, M. H. Sarmadi, and C. Vafa, Nucl. Phys. B288, 551 (1987).

[3] H. Kawai, D. C. Lewellen, and S.-H. H. Tye, Phys. Rev. Lett. 57, 1832 (1986), 58, 429E (1987); Nucl. Phys. B288, 1 (1987); I. Antoniadis, C. P. Bachas, and C. Kounnas, ibid. B289, 87 (1987).

[4] P. Ginsparg, Nucl. Phys. B295, 153 (1988); see also R. Dijkgraaf, C. Vafa, E. Verlinde, and H. Verlinde, Commun. Math. Phys. 123, 485 (1989).

[5] Z. S. Li and C. S. Lam, Int. J. Mod. Phys. A7, 5739 (1992).

[6] Z. Kakushadze and S.-H. H. Tye, this issue, Phys. Rev. D 54, 7520 (1996).

[7] K. Narain, Phys. Lett. 169B, 41 (1986). K. Narain, M. H.
Sarmadi, and E. Witten, Nucl. Phys. B279, 369 (1986).

[8] Z. Kakushadze and S.-H. H. Tye, Phys. Rev. Lett. 77, 2612 (1996).

[9] H. Kawai, D. C. Lewellen, J. A. Schwartz, and S.-H. H. Tye, Nucl. Phys. B299, 431 (1988).

[10] H. Kawai, D. C. Lewellen, and S.-H. H. Tye, Phys. Rev. D 34, 3794 (1986); L. Dixon and J. Harvey, Nucl. Phys. B274, 93 (1986).

[11] P. Ginsparg and C. Vafa, Nucl. Phys. B289, 414 (1987).

[12] D. S. Freed and C. Vafa, Commun. Math. Phys. 110, 349 (1987).

[13] I. Antoniadis and C. Bachas, Nucl. Phys. B298, 586 (1988); H. Dreiner, J. L. Lopez, D. V. Nanopoulos, and D. B. Reiss, ibid. B320, 401 (1989); G. Cleaver, ibid. B456, 219 (1995). 